Ann. Scient. Éc. Norm. Sup.,

$4^{\mathrm{e}}$ série, t. 40, 2007, p. 741 à 764.

\title{
SUR LE GROUPE D’AUTOMORPHISMES DES GÉOMÉTRIES PARABOLIQUES DE RANG 1
}

\author{
PAR CHARLES FRANCES
}

RÉSUMÉ. - Dans cet article, nous prouvons le résultat suivant, qui englobe le théorème de Ferrand-Obata sur le groupe conforme d'une variété riemannienne, et le théorème de Schoen-Webster sur le groupe des automorphismes d'une structure $C R$ strictement pseudo-convexe : soit $M$ une variété connexe munie d'une géométrie de Cartan régulière modelée sur le bord $\mathbf{X}=\partial \mathbf{H}_{\mathbb{K}}^{d}$ de l'espace hyperbolique de dimension $d \geqslant 2$ sur $\mathbb{K}, \mathbb{K}$ pouvant être $\mathbb{R}, \mathbb{C}, \mathbb{H}$ ou l'algèbre des octonions $\mathbb{O}$. Si le groupe d'automorphismes de $M$ n'agit pas proprement sur $M$, alors $M$ est isomorphe, comme géométrie de Cartan, à l'espace $\mathbf{X}$, ou à $\mathbf{X}$ privé d'un point.

(c) 2007 Elsevier Masson SAS

ABSTRACT. - The aim of this article is to prove the following result, which generalizes the Ferrand-Obata theorem, concerning the conformal group of a Riemannian manifold, and the Schoen-Webster theorem about the automorphism group of a strictly pseudo-convex $C R$ structure: let $M$ be a connected manifold endowed with a regular Cartan geometry, modelled on the boundary $\mathbf{X}=\partial \mathbf{H}_{\mathbb{K}}^{d}$ of the hyperbolic space of dimension $d \geqslant 2$ over $\mathbb{K}, \mathbb{K}$ being $\mathbb{R}, \mathbb{C}, \mathbb{H}$ or the octonions $\mathbb{O}$. If the automorphism group of $M$ does not act properly on $M$, then $M$ is isomorphic, as a Cartan geometry, to $\mathbf{X}$, or $\mathbf{X}$ minus a point.

(c) 2007 Elsevier Masson SAS

\section{Introduction}

Au début des années soixante-dix, les travaux conjugués de différents auteurs sur la conjecture de Lichnerowicz en géométrie conforme débouchaient sur le résultat suivant, qui transforme la conjecture en théorème :

THÉORÈME (Ferrand-Obata [21,8,9]). - Soit $(M, g)$ une variété riemannienne de dimension $n \geqslant 2$. Si le groupe des transformations conformes de $(M, g)$ n'agit pas proprement sur $M$, alors $(M, g)$ est conformément équivalente à la sphère conforme standard si $M$ est compacte, ou à l'espace euclidien sinon.

Rappelons ici que l'action d'un groupe $G$ par homéomorphismes sur une variété $M$ est propre si, pour tout compact $K \subset M$, l'ensemble :

$$
G_{K}=\{g \in G \mid g(K) \cap K \neq \emptyset\}
$$

est d'adhérence compacte dans le groupe des homéomorphismes de $M$, muni de la topologie compacte-ouverte. On ne suppose pas a priori $G$ fermé dans le groupe des homéomorphismes.

Pour rendre justice au travail de J. Ferrand, précisons que c'est à elle que l'on doit l'énoncé et la preuve définitive du théorème ci-dessus (notamment le cas non compact), la preuve de M. Obata 
ne traitant que du cas plus restrictif où $M$ est compacte, et surtout où la composante neutre du groupe conforme n'agit pas proprement. Il est à noter qu'une vingtaine d'années s'écoulèrent entre la version initiale du théorème dans le cas compact ([21,8]), et la preuve définitive (voir [9]).

Une autre preuve du théorème de Ferrand-Obata a été proposée par R. Schoen dans [23]. Ses méthodes donnent en outre un résultat analogue pour les structures $C R$, étendant ainsi des travaux antérieurs de S.M. Webster.

THÉORÈME (Schoen-Webster [23,29]). - Soit $M^{2 n+1}, n \geqslant 1$, une variété munie d'une structure $C R$ strictement pseudo-convexe. Si le groupe des automorphismes $C R$ de $M$ n'agit pas proprement sur $M$, alors $M$ est $C R$-difféomorphe à la sphère $C R$ standard si $M$ est compacte, ou au groupe de Heisenberg, muni de sa structure standard, sinon.

\subsection{Géométries de Cartan}

Bien que les méthodes utilisées par J. Ferrand et R. Schoen soient totalement différentes, la similitude entre les énoncés des théorèmes précédents laisse soupçonner qu'ils sont deux facettes d'un phénomène plus général. En termes de structures géométriques, il existe un concept qui unifie, en dimension $n \geqslant 3$, les structures conformes et les structures $C R$ strictement pseudoconvexes : celui de géométrie de Cartan.

Une géométrie de Cartan est la donnée d'une variété infinitésimalement modelée sur un espace homogène $\mathbf{X}=G / P$, où $G$ est un groupe de Lie, et $P$ est un sous-groupe fermé de $G$. Formellement, une géométrie de Cartan sur une variété $M$, modelée sur l'espace homogène $\mathbf{X}=G / P$, est la donnée :

(i) d'un $P$-fibré principal $B \rightarrow M$ au-dessus de $M$,

(ii) d'une 1-forme $\omega$ sur $B$ à valeurs dans l'algèbre de Lie $\mathfrak{g}$, appelée connexion de Cartan, et satisfaisant les conditions suivantes :

- en tout point $p \in B, \omega_{p}$ réalise un isomorphisme de $T_{p} B$ sur $\mathfrak{g}$,

- si $X^{\dagger}$ est le champ de vecteurs sur $B$ issu de l'action par multiplication à droite d'un groupe à 1 paramètre $t \mapsto \operatorname{Exp}_{G}(t X)$ de $P$, alors $\omega\left(X^{\dagger}\right)=X$,

- pour tout $a \in P, R_{a}{ }^{*} \omega=A d\left(a^{-1}\right) \omega\left(R_{a}\right.$ indiquant ici l'action à droite de $\left.a \operatorname{sur} B\right)$.

Une géométrie de Cartan sur une variété $M$ sera notée $(M, B, \omega)$.

De nombreuses structures géométriques classiques s'interprètent en termes de géométries de Cartan. Les plus célèbres sont les métriques riemanniennes (resp. pseudo-riemanniennes de signature $(p, q)$ ). Dans ce cas, l'espace $\mathbf{X}$ est l'espace euclidien (resp. l'espace de Minkowski de signature $(p, q)), G$ est le groupe des isométries $S O(n) \ltimes \mathbb{R}^{n}$ (resp. $S O(p, q) \ltimes \mathbb{R}^{n}$ ), et $P$ le sous-groupe linéaire $S O(n)$ (resp. $S O(p, q)$ ).

Étant donnée la définition assez peu intuitive d'une géométrie de Cartan, deux problèmes intéressants, et délicats, se posent d'emblée. Le premier consiste à interpréter la donnée d'une connexion de Cartan $\omega$ sur un fibré principal $B$ au-dessus de $M$, directement en termes de structure géométrique sur $M$. On sait donner une telle interprétation dans de nombreux cas intéressants (voir [3,27,20], et les autres références données dans ces articles).

Le second est de savoir si une structure géométrique donnée sur une variété $M$ définit canoniquement une géométrie de Cartan. C'est généralement un problème difficile, que l'on appelle le problème d'équivalence. Il a été résolu par E. Cartan lui-même pour les structures conformes riemanniennes de dimension $n \geqslant 3$, ainsi que pour les structures $C R$ strictement pseudo-convexes (en dimension 3, voir [4]. Pour la dimension $\geqslant 3$, voir [7] ainsi que [26]. Le cas conforme est détaillé dans [16,24]). Autrement dit, si $M$ (de dimension $n \geqslant 3$ ) est munie d'une classe conforme de métriques riemanniennes (resp. d'une structure $C R$ strictement pseudo-convexe), alors on sait construire un $P$-fibré principal $B$ au-dessus de $M$, et une connexion de Cartan $\omega$ sur $B$. Dans le cas conforme (resp. $C R$ strictement pseudo-convexe), l'espace modèle $\mathbf{X}$

$4^{\mathrm{e}}$ SÉRIE - TOME $40-2007-\mathrm{N}^{\circ} 5$ 
est la sphère conforme $\mathbf{S}^{n}=\partial \mathbf{H}_{\mathbb{R}}^{n+1}$ (resp. la sphère $C R \mathbf{S}^{2 n+1}=\partial \mathbf{H}_{\mathbb{C}}^{n+1}$ ), le groupe $G$ est le groupe de Möbius $P O(1, n+1)$ (resp. le groupe $P U(1, n+1)$ ), et $P$ est un groupe parabolique : le stabilisateur d'un point de $\mathbf{X}$. D'autre part, sous certaines conditions de normalisation, la connexion de Cartan $\omega$ est définie de manière unique par la structure sur $M$. Ainsi, le groupe des difféomorphismes conformes (resp. des difféomorphismes $C R$ ) agit sur $B$ en préservant $\omega$.

Pour une géométrie de Cartan quelconque $(M, B, \omega)$, on définit $A u t(B, \omega)$ comme l'ensemble des difféomorphismes $\phi$ de $B$, de classe $C^{1}$, vérifiant $\phi^{*} \omega=\omega$. Tout $\phi$ de $A u t(B, \omega)$ commute avec l'action à droite de $P$ sur $B$. En particulier $\phi$ permute les fibres de $B$ et induit un difféomorphisme $\bar{\phi}$ de $M$. L'ensemble de ces difféomorphismes est noté $A u t(M, \omega)$.

On définit également une notion d'équivalence géométrique pour deux géométries de Cartan $(M, B, \omega)$ et $\left(N, B^{\prime}, \omega^{\prime}\right)$ modelées sur un même espace $\mathbf{X}=G / P$. On dit que $M$ et $N$ sont géométriquement isomorphes s'il existe un difféomorphisme de fibrés $\phi: B \rightarrow B^{\prime}$ tel que $\phi^{*} \omega^{\prime}=\omega$.

\section{2. Énoncé des résultats}

Nous nous proposons de généraliser les théorèmes de Ferrand-Obata et Schoen-Webster à toutes les géométries de Cartan modelées sur les espaces $\mathbf{X}=G / P$, où $G$ est un groupe de Lie simple de rang 1 , de type non compact, de centre fini, et $P$ un sous-groupe parabolique de $G$. Ces espaces $\mathbf{X}$ sont les bords des différents espaces hyperboliques $\mathbf{H}_{\mathbb{K}}^{d}, \mathbb{K}$ pouvant être le corps des réels $\mathbb{R}$, des complexes $\mathbb{C}$, des quaternions $\mathbb{H}$, ou l'algèbre des octonions $\mathbb{O}$. On supposera $d \geqslant 2$ si $\mathbb{K}=\mathbb{R}$, et $d \geqslant 1$ sinon. Dans le cas des octonions $\mathbb{O}$, la dimension $d$ est nécessairement 2 .

Implicitement, lorsque l'on parlera de géométrie de Cartan modelée sur $\partial \mathbf{H}_{\mathbb{K}}^{d}$, on verra toujours $\partial \mathbf{H}_{\mathbb{K}}^{d}$ comme l'espace homogène $G / P$, où $G=I s o\left(\mathbf{H}_{\mathbb{K}}^{d}\right)$, et $P$ est le stabilisateur dans Iso $\left(\mathbf{H}_{\mathbb{K}}^{d}\right)$, d'un point de $\partial \mathbf{H}_{\mathbb{K}}^{d}$. Rappelons que les groupes $G$ qui interviennent sont :

- $G=P O(1, d), d \geqslant 2$ pour $\mathbb{K}=\mathbb{R}$. L'espace $\mathbf{X}=\partial \mathbf{H}_{\mathbb{R}}^{d}$ est une sphère $\mathbf{S}^{d-1}$.

- $G=P U(1, d), d \geqslant 1$ pour $\mathbb{K}=\mathbb{C}$. L'espace $\mathbf{X}=\partial \mathbf{H}_{\mathbb{C}}^{d}$ est une sphère $\mathbf{S}^{2 d-1}$.

- $G=S p(1, d), d \geqslant 1$ pour $\mathbb{K}=\mathbb{H}$. L'espace $\mathbf{X}=\partial \mathbf{H}_{\mathbb{H}}^{d}$ est une sphère $\mathbf{S}^{4 d-1}$.

- $G=F_{4}^{-20}$ lorsque $\mathbb{K}=\mathbb{O}$. L'espace $\mathbf{X}=\partial \mathbf{H}_{\mathbb{O}}^{2}$ est une sphère $\mathbf{S}^{15}$.

On peut maintenant énoncer notre résultat principal :

THÉORÈme 1. - Soit $(M, B, \omega)$ une géométrie de Cartan modelée sur le bord $\mathbf{X}=\partial \mathbf{H}_{\mathbb{K}}^{d}$ d'un espace hyperbolique de dimension d sur $\mathbb{K}=\mathbb{R}, \mathbb{C}, \mathbb{H}$ ou $\mathbb{O}$. On suppose que $M$ est connexe et que la connexion $\omega$ est régulière. Si Aut $(M, \omega)$ n'agit pas proprement sur $M$, alors $M$ est géométriquement isomorphe à $\mathrm{X}$ si $M$ est compacte, ou à $\mathrm{X}$ privé d'un point sinon.

Précisons que nous aurons besoin, au cours de la preuve, que la connexion $\omega$ soit de classe $C^{1}$. Par exemple, dans le cas conforme riemannien, cela nécessite que les métriques de la classe conforme soient de classe $C^{2}$.

L'hypothèse de régularité est une hypothèse technique sur la courbure de la connexion de Cartan (vérifiée dans la plupart des cas intéressants) que nous introduirons en section 3.2.1.

Discutons à présent le champ d'application du théorème 1. Les conclusions du théorème peuvent s'appliquer à toute structure géométrique sur une variété $M$, pour laquelle on a résolu le problème d'équivalence, autrement dit toute structure à partir de laquelle on sait définir canoniquement une géométrie de Cartan modelée sur un des espaces $\mathbf{X}=\partial \mathbf{H}_{\mathbb{K}}^{d}$, telle que la connexion de Cartan "canonique" soit régulière. Au vu des travaux existants sur le problème d'équivalence, le théorème $1 \mathrm{~s}$ 'applique donc aux structures conformes riemanniennes et aux structures $C R$ strictement pseudo-convexes en dimension $n \geqslant 3$ (il donne ainsi une preuve unifiée des théorèmes de Ferrand-Obata et Schoen-Webster), ainsi qu'aux structures presque $C R$ partiellement intégrables $([3,20,27])$, et également aux structures de contact quaternioniennes et octoniennes introduites par O. Biquard ([1], voir également [2]). 
Remarquons que, malgré les hypothèses faites sur la dimension $d$, le théorème 1 ne s'applique pas aux structures conformes sur les surfaces. En effet, en dimension 2, la donnée d'une structure conforme ne définit pas une connexion de Cartan canonique. On pourra consulter [25] pour une interprétation de ce fait en termes de $G$-structures.

\subsection{Idées de la preuve et organisation de l'article}

La preuve du théorème 1 repose essentiellement sur la compréhension de la dynamique des suites d'automorphismes d'une variété $M$, munie d'une géométrie de Cartan modelée sur $\mathbf{X}=\partial \mathbf{H}_{\mathbb{K}}^{d}$. Le point central sera de montrer que, si une suite $\left(f_{k}\right)$ de $\operatorname{Aut}(M, \omega)$ n'agit pas proprement sur $M$, alors elle a la propriété $(P)$ suivante :

$(P)$ : il existe un ouvert non vide $U \subset M$ qui est contracté sur un point sous l'action de la suite $\left(f_{k}\right)$.

C'est une propriété fondamentale car on peut montrer qu'elle implique que la géométrie est alors plate sur l'ouvert $U$.

En fait, J. Ferrand et R. Schoen montrent également la propriété $(P)$ pour les suites d'applications conformes (resp. de difféomorphismes $C R$ ) qui n'agissent pas propement. Il faut noter qu'ils arrivent à cette conclusion par des méthodes d'analyse (J. Ferrand déclare d'ailleurs dans l'introduction de [9] "In fact, [the theorem of Ferrand-Obata] is not actually concerning the theory of Lie groups and may be considered as a mere theorem of Analysis".)

L'approche que nous développons pour arriver à la propriété $(P)$ est au contraire complètement géométrique. On commence par rappeler que la propriété $(P)$ est typique des suites de $G=I s o\left(\mathbf{H}_{\mathbb{K}}^{d}\right)$ qui tendent vers l'infini, lorsqu'elles agissent sur le bord $\mathbf{X}=\partial \mathbf{H}_{\mathbb{K}}^{d}$. C'est ce que l'on appelle une dynamique "Nord-Sud", ou "de convergence" (voir section 2.4.2). L'idée principale dans la démonstration du théorème 1 va être de montrer comment la connexion de Cartan permet de relier la dynamique des suites de $\operatorname{Aut}(M, \omega)$ et celle des suites de $G$ sur X. Pour fixer les idées, commençons par dire quelques mots du cas simple où l'on sait que la géométrie de Car$\tan \operatorname{sur} M$ est plate. On est alors en présence de ce que l'on appelle une $(G, \mathbf{X})$-structure sur $M$. On peut dans ce cas définir une application développante $\delta: \tilde{M} \rightarrow \mathbf{X}$, qui est une immersion, et un morphisme $\rho: \operatorname{Aut}(\tilde{M}, \tilde{\omega}) \rightarrow G$, satisfaisant la relation d'équivariance : $\delta \circ f=\rho(f) \circ \delta$, pour tout $f \in \operatorname{Aut}(\tilde{M}, \tilde{\omega})$ (on renvoie à [17] pour un des premiers exemples d'utilisation du développement, dans le cas conforme. Pour des résultats généraux sur les $(G, X)$-structures, voir [28]). Cette relation d'équivariance permet de reconstituer, au moins localement, la dynamique d'une suite $\left(f_{k}\right)$ de $A u t(\tilde{M}, \tilde{\omega})$ à partir de la dynamique de $\rho\left(f_{k}\right)$ sur $\mathbf{X}$ (voir par exemple $[18,12]$ ).

Bien entendu, la géométrie de Cartan considérée sur $M$ n'est pas plate a priori. Bien que les outils classiques d'étude des $(G, \mathbf{X})$-structures ne soient plus valables dans ce cas, il subsiste quelques traces du schéma précédent. Fixons-nous $x_{0} \in M, \hat{x}_{0} \in B$ au-dessus de $x_{0}$, et $o \in \mathbf{X}$. La connexion de Cartan permet de définir une application développante, notée $\mathcal{D}_{x_{0}}^{\hat{x}_{0}}$, qui va cette fois-ci de l'espace des courbes de $M$ passant par $x_{0}$ dans celui des courbes de $\mathbf{X}$ passant par $o$. Ce procédé, par ailleurs classique, sera détaillé en section 3.3.

D'autre part, si $\left(f_{k}\right)$ est une suite de $\operatorname{Aut}(M, \omega)$ qui, pour simplifier, fixe $x_{0}$, il est possible, comme nous le verrons en section 5.2, de lui associer une suite d'holonomie $\left(b_{k}\right)$ de $G$, telle que tous les $b_{k}$ fixent $o$ et satisfont la relation d'équivariance :

$$
\mathcal{D}_{x_{0}}^{\hat{x}_{0}} \circ f_{k}=b_{k} \circ \mathcal{D}_{x_{0}}^{\hat{x}_{0}} .
$$

Il s'agit ici d'un principe général, qui pourra probablement servir à l'étude dynamique des automorphismes d'autres géométries de Cartan. La relation (1) est fondamentale, puisqu'elle permet de faire le lien entre l'action de $\left(b_{k}\right)$ sur les courbes de $\mathbf{X}$ passant par $o$ et l'action de $\left(f_{k}\right)$

$4^{\text {e }}$ SÉRIE - TOME $40-2007-\mathrm{N}^{\circ} 5$ 
sur les courbes de $M$ passant par $x_{0}$. Bien sûr, l'espace des courbes de $\mathbf{X}$ (resp. de $M$ ) passant par $o$ (resp. $x_{0}$ ) étant beaucoup trop gros, on va se restreindre à l'action sur une famille de courbes, privilégiées pour la géométrie considérée : les géodésiques de la géométrie de Cartan. Nous définissons ces géodésiques en section 4 . On retrouve les géodésiques conformes dans le cas où $\mathbf{X}=\partial \mathbf{H}_{\mathbb{R}}^{d}$ et les "chaînes" d'E. Cartan pour la géométrie $C R$, dans le cas où $\mathbf{X}=\partial \mathbf{H}_{\mathbb{R}}^{d}$.

L'idée générale pour obtenir la propriété $(P)$ est que la dynamique de type "Nord-Sud" de la suite $\left(b_{k}\right)$ va se traduire dans l'action de $\left(b_{k}\right)$ sur l'espace des géodésiques passant par $o$ (c'est l'objet de la section 2.4.3), et que la relation (1) va transmettre cette propriété à l'action de la suite $\left(f_{k}\right)$ sur les géodésiques passant par $x_{0}$. On obtient alors la dynamique de $\left(f_{k}\right)$ au voisinage de $x_{0}$ par projection. Remarquons que le comportement dynamique de type NordSud est caractéristique du rang 1, et c'est essentiellement pour cela que l'on n'a pas d'analogue au théorème 1 en rang supérieur (pour les structures conformes lorentziennes, par exemple, voir [11]).

Comme nous l'avons dit plus haut, une fois la propriété $(P)$ établie, on obtient un ouvert $U \subset M$ sur lequel la géométrie de Cartan est plate. Le théorème 1 s'obtient en identifiant l'ouvert $U$ à $M$, ou $M$ privée d'un point. Nous arrivons à cette conclusion grâce à un résultat de rigidité pour les plongements géométriques de certaines géométries de Cartan. Il s'agit du théorème 4 , qui est énoncé à la fin de la section 6, et dont la preuve fait l'objet de la dernière partie de l'article.

\section{Géométrie des espaces modèles $\mathrm{X}$}

\subsection{Préliminaires algébriques}

La plupart des préliminaires qui suivent sont très clairement exposés dans [15]. Soit $G$ un groupe de Lie simple, de centre fini, et de rang réel 1. On appelle $\mathfrak{g}$ son algèbre de Lie. On fait le choix d'une involution de Cartan $\theta$ sur $\mathfrak{g}$. Cette involution donne une décomposition dite de Cartan $: \mathfrak{g}=\mathfrak{k}_{\theta} \oplus \mathfrak{p}_{\theta}$. On choisit $\mathfrak{a}$ une sous-algèbre abélienne maximale de $\mathfrak{p}_{\theta}$, qui est de dimension 1 , vu notre hypothèse sur le rang de $G$. Pour toute la suite, on choisit $X_{0} \neq 0$ dans $\mathfrak{a}$, tel que $\alpha\left(X_{0}\right)=1$, et on note $A$ le groupe à un paramètre $a^{t}=\operatorname{Exp}_{G}\left(t X_{0}\right)$. Notons $\Delta$ l'ensemble des racines pour la représentation adjointe de $\mathfrak{a}$ sur $\mathfrak{g}$. Pour $\lambda \in \Delta$, on pose $\mathfrak{g}_{\lambda}=\left\{v \in \mathfrak{g} \mid A d\left(a^{t}\right) v=e^{t \lambda\left(X_{0}\right)} v\right\}$. Comme $G$ est de rang 1 , il y a seulement deux possibilités pour $\Delta$ :

- $\Delta=\{-\alpha,+\alpha\}($ cas $\mathfrak{g}=\mathfrak{s o}(1, n), n \geqslant 2$, ou $\mathfrak{s u}(1,1)$ ou $\mathfrak{s p}(1,1))$,

$-\Delta=\{-2 \alpha,-\alpha,+\alpha,+2 \alpha\}$ (tous les autres cas).

Dans les deux cas, l'algèbre de Lie $\mathfrak{g}$ admet la décomposition $\mathfrak{g}=\mathfrak{n}^{-} \oplus \mathfrak{a} \oplus \mathfrak{m} \oplus \mathfrak{n}^{+}$. L'algèbre $\mathfrak{l}=\mathfrak{a} \oplus \mathfrak{m}$ est le centralisateur de $\mathfrak{a}$ dans $\mathfrak{g}$. Elle est stable par l'involution de Cartan $\theta$ et $\mathfrak{a}$ correspond au sous-espace propre de $\mathfrak{l}$ associé à la valeur propre -1 alors que $\mathfrak{m}$ est le sousespace des points fixes de $\theta$ dans $\mathfrak{l}$.

On écrit $\mathfrak{n}^{+}=\mathfrak{n}_{1}^{+} \oplus \mathfrak{z}^{+}\left(\right.$resp. $\left.\mathfrak{n}^{-}=\mathfrak{z}^{-} \oplus \mathfrak{n}_{1}^{-}\right)$où $\mathfrak{z}^{+}\left(\right.$resp. $\left.\mathfrak{z}^{-}\right)$est le centre de $\mathfrak{n}^{+}\left(\right.$resp. $\left.\mathfrak{n}^{-}\right)$.

Précisément, si $\Delta=\{-\alpha,+\alpha\}$, on a tout simplement $\mathfrak{z}^{+}=\mathfrak{n}^{+}=\mathfrak{g}_{+\alpha}\left(\right.$ resp. $\left.\mathfrak{z}^{-}=\mathfrak{n}^{-}=\mathfrak{g}_{-\alpha}\right)$, et $\mathfrak{n}_{1}^{+}=\mathfrak{n}_{1}^{-}=\{0\}$.

Lorsque $\Delta=\{-2 \alpha,-\alpha,+\alpha,+2 \alpha\}$, on a $\mathfrak{n}^{+}=\mathfrak{g}_{+\alpha} \oplus \mathfrak{g}_{+2 \alpha}$ (resp. $\mathfrak{n}^{-}=\mathfrak{g}_{-2 \alpha} \oplus \mathfrak{g}_{-\alpha}$ ), $\mathfrak{z}^{+}=\mathfrak{g}_{+2 \alpha}\left(\right.$ resp. $\left.\mathfrak{z}^{-}=\mathfrak{g}_{-2 \alpha}\right)$ ) et $\mathfrak{n}_{1}^{+}=\mathfrak{g}_{+\alpha}\left(\right.$ resp. $\left.\mathfrak{n}_{1}^{-}=\mathfrak{g}_{-\alpha}\right)$.

Les deux algèbres de Lie $\mathfrak{n}^{+}$et $\mathfrak{n}^{-}$sont nilpotentes. L'application $\operatorname{Exp}_{G}$ réalise un difféomorphisme de $\mathfrak{n}^{+}$( resp. $\mathfrak{n}^{-}$) sur un sous-groupe de Lie fermé $N^{+} \subset G$ (resp. $N^{-} \subset G$ ).

Le groupe parabolique $P$ est le sous-groupe fermé de $G$ dont l'algèbre de Lie est $\mathfrak{p}=$ $\mathfrak{a} \oplus \mathfrak{m} \oplus \mathfrak{n}^{+}$. On notera $\pi_{X}$ la projection $G \rightarrow G / P=\mathbf{X}$. Comme nous l'avons déjà dit, l'espace $\mathbf{X}$ est difféomorphe à une sphère. 
Rappelons également la décomposition de Langlands $P=M A N^{+}$pour le groupe $P$, où $M$ et $A$ sont les sous-groupes de $G$, ayant pour algèbres de Lie respectives $\mathfrak{m}$ et $\mathfrak{a}$. On notera $L=M A$.

\subsection{Cartes}

- Atlas : on appelle $o$ la projection de $P$ sur $G / P$. Comme le groupe $G$ est de rang réel 1, son groupe de Weyl est un groupe à deux éléments $\{e, w\}$. L'élément $w$ agit sur $\mathbf{X}$ par une involution, et envoie $o$ sur un point $\nu \in \mathbf{X}$. Les points $o$ et $\nu$ sont laissés fixes par le groupe $L$. D'autre part, $w N^{+} w=N^{-}$. La décomposition de Bruhat (voir par exemple [15]) s'écrit alors $G=P \cup P w P$. Autrement dit, si l'on appelle $\Omega_{o}$ (resp. $\Omega_{\nu}$ ) l'orbite de $o$ sous l'action de $N^{-}$(resp. l'orbite de $\nu$ sous l'action de $N^{+}$), la variété $\mathbf{X}$ peut s'écrire comme l'union : $\mathbf{X}=\{o\} \cup \Omega_{\nu}=\{\nu\} \cup \Omega_{o}$.

Cela fournit un atlas à deux cartes sur $\mathbf{X}:$ l'application $s^{-}: \mathfrak{n}^{-} \rightarrow \mathbf{X}$ définie par $s^{-}(u)=$ $\operatorname{Exp}_{G}(u) . o$, qui réalise un difféomorphisme de $\mathfrak{n}^{-}$sur l'ouvert $\Omega_{o}$. On a de même la carte $s^{+}: \mathfrak{n}^{+} \rightarrow \mathbf{X}$ définie par $s^{+}(u)=\operatorname{Exp}_{G}(u) . \nu$, qui réalise un difféomorphisme de $\mathfrak{n}^{+}$sur l'ouvert $\Omega_{\nu}$.

- Métriques auxiliaires : on munit $\mathfrak{g}$ d'un produit scalaire noté \langle\rangle$_{\mathfrak{g}}$ invariant par l'involution de Cartan $\theta$, et l'on note $\|$.$\| la norme associée sur \mathfrak{g}$. On étend ce produit scalaire en une métrique riemannienne $\rho_{G}$ sur $G$, invariante à gauche. Celle-ci induit à son tour sur $N^{+}$ et $N^{-}$deux métriques riemanniennes invariantes à gauche $\rho^{+}$et $\rho^{-}$, et l'on transporte ces métriques sur $\Omega_{\nu}$ et $\Omega_{o}$. On obtient ainsi deux métriques riemanniennes sur $\Omega_{\nu}$ et $\Omega_{o}$ (toujours notées $\rho^{+}$et $\rho^{-}$), pour lesquelles $N^{+}$et $N^{-}$agissent par isométries.

- Changements de cartes: On pose $s_{-}^{+}=\left(s^{+}\right)^{-1} \circ s^{-} ; s_{-}^{+}$va donc de $\mathfrak{n}^{-} \backslash\{0\}$ dans $\mathfrak{n}^{+} \backslash\{0\}$. On va donner une formule pour la restriction de $s_{-}^{+}$à $\mathfrak{z}^{-} \backslash\{0\}$.

Soit $u \in \mathfrak{z}^{-}, u \neq 0$. On note $w=[u, \theta u]$. Sachant qu'il existe $\delta \in \Delta$ tel que $\mathfrak{z}^{-}=\mathfrak{g}_{\delta}$, on voit que $\mathbb{R} . u \oplus \mathbb{R} . w \oplus \mathbb{R} . \theta u$ est une sous-algèbre de Lie de $\mathfrak{g}$ isomorphe à $\mathfrak{s l}(2, \mathbb{R})$. En fait, il existe une normalisation $u^{\prime}=\lambda u, w^{\prime}=\mu w$, et $v^{\prime}=\theta u$, telle que $\left[w^{\prime}, u^{\prime}\right]=2 u^{\prime},\left[w^{\prime}, v^{\prime}\right]=-2 v^{\prime}$ et $\left[u^{\prime}, v^{\prime}\right]=w^{\prime}$. Par la formule de la projection stéréographique en dimension 1, on obtient qu'il existe un réel $a_{u}$ (qui en fait ne dépend que de la direction de $u$ ) tel que :

$$
s_{-}^{+}(u)=a_{u} \cdot \frac{\theta u}{\|u\|^{2}} .
$$

\subsection{Géodésiques sur $X$}

Commençons par introduire quelques notations. Pour tout $u \in \mathfrak{g}$, on appelle $u^{*}$ la courbe de $[0,1]$ dans $\mathbf{X}$ définie par $u^{*}(t)=\pi_{X} \circ \operatorname{Exp}_{G}(t u)$. Par $[u]$, on désignera le support géométrique de la courbe $u^{*}$.

\subsubsection{Géodésiques paramétrées}

On définit le sous-ensemble $\mathbf{Q} \subset \mathfrak{g}$ suivant :

$$
\mathbf{Q}=\left\{v \in \mathfrak{g} \mid v=A d(b) . u, u \in \mathfrak{z}^{-}, b \in P\right\} .
$$

DÉFINITION 1 (Géodésiques paramétrées et segments géodésiques). - On appelle géodésique de $\mathbf{X}$ toute courbe de $I$ dans $\mathbf{X}$, où $I$ est un intervalle contenant 0 , qui est de la forme $t \rightarrow g . \pi_{X} \circ \operatorname{Exp}_{G}(t u)$ avec $u \in \mathbf{Q}$ et $g \in G$. Lorsque $I=\mathbb{R}$, on parle de géodésique maximale.

- Pour tout $u \in \mathbf{Q}$, la courbe $u^{*}$ s'appelle le segment géodésique paramétré issu de $o$ associé à $u$.

$4^{\text {e }}$ SÉRIE - TOME $40-2007-\mathrm{N}^{\circ} 5$ 
- On dit que $[u]$ est le segment géodésique (non paramétré) issu de $o$, associé à $u$. L'espace des segments géodésiques issus de $o$ est noté $[\mathbf{Q}]$.

- On désignera par $\dot{\mathbf{Q}}$ (resp. $[\dot{\mathbf{Q}}]$ ) l'espace $\mathbf{Q}$ privé du point $0_{\mathfrak{g}}$ (resp. l'espace $[\mathbf{Q}]$ privé du segment trivial $[o])$.

Dans toute la suite, nous munirons $[\mathbf{Q}]$ de la topologie de Hausdorff sur les fermés de $\mathbf{X}$.

\subsubsection{Propriétés fondamentales de $[\mathrm{Q}]$}

Notre première tâche va être de décrire $[\mathbf{Q}]$ plus en détail, et de mieux comprendre l'action de $P \operatorname{sur}[\mathbf{Q}]$.

Lemme 1. - Il existe un morphisme $\rho: P \rightarrow A f f\left(\mathfrak{n}^{+}\right)$tel que pour tout $b \in P: b \circ s^{+}=$ $s^{+} \circ \rho(b)$.

On va étudier séparément l'action des différentes composantes de la décomposition de Langlands.

- Action de L.

Soient $l$ un élément de $L$, et $u \in \mathfrak{n}^{+}$. On pose $n^{+}=\operatorname{Exp}_{G}(u)$ et $x=n^{+} . \nu$. Alors $l . x=$ $A d(l)\left(n^{+}\right) . l . \nu$, et comme $l . \nu=\nu, l . x=s^{+}(A d(l) . u)$. Ainsi, $\rho(l)=A d(l)_{\mid \mathfrak{n}^{+}}: l^{\prime}$ 'action de $L$ lue dans la carte $s^{+}$est simplement l'action adjointe.

- Action de $\mathrm{N}^{+}$.

Soient $n_{0}^{+}=\operatorname{Exp}\left(u_{0}\right)$ et $n^{+}=\operatorname{Exp}(u)$ deux éléments de $N^{+}$. Comme $\mathfrak{n}^{+}$est nilpotente d'ordre 1 ou 2, la formule de Campbell-Hausdorff assure que $n_{0}^{+} n^{+}=\operatorname{Exp}\left(u_{0}+u+\right.$ $\left.\frac{1}{2} a d\left(u_{0}\right)(u)\right)$. On obtient donc que $n_{0}^{+} \cdot s^{+}(u)=s^{+}\left(\left(I d+\frac{1}{2} a d\left(u_{0}\right)\right) \cdot u+u_{0}\right)$, soit encore :

$$
\rho\left(n_{0}^{+}\right): u \mapsto\left(\operatorname{Id}+\frac{1}{2} a d\left(u_{0}\right)\right) \cdot u+u_{0} .
$$

Remarque 1.- Si $u \in \mathfrak{z}^{+}$, on a juste $\rho\left(n_{0}^{+}\right) \cdot u=u+u_{0}$, c'est-à-dire que $\rho\left(N^{+}\right)$agit par translations sur $\mathfrak{z}^{+}$.

Pour toute demi-droite $\alpha$ de $\mathfrak{n}^{+}$, il existe un unique point $x \in \mathfrak{n}^{+}$et un unique vecteur $v \in \mathfrak{n}^{+}$ de norme 1 pour $\|$.$\| , tels que \alpha=\left\{y \in \mathfrak{n}^{+} \mid y=x+t v, t \in \mathbb{R}_{+}\right\}$. Ainsi, si $S_{\mathfrak{n}^{+}}$(resp. $S_{\mathfrak{z}^{+}}$) désigne la sphère unité de $\mathfrak{n}^{+}$(resp. $\mathfrak{z}^{+}$) pour la norme $\|$.$\| , l'espace des demi-droites de \mathfrak{n}^{+}$ (resp. des demi-droites dont la direction est dans $\mathfrak{z}^{+}$) s'identifie au produit $\mathfrak{n}^{+} \times S_{\mathfrak{n}^{+}}$(resp. $\mathfrak{n}^{+} \times S_{\mathfrak{z}^{+}}$). Par la suite, on notera une telle demi-droite $[x, u)$, avec $x \in \mathfrak{n}^{+}$et $u \in S_{\mathfrak{n}^{+}}$(resp. $u \in S_{\mathfrak{z}^{+}}$.

Remarque 2. - Le groupe $\rho(P)$ laisse stable l'ensemble des demi-droites affines de $\mathfrak{n}^{+}$dont la direction est dans $\mathfrak{z}^{+}$.

Proposition 1.- (i) Via l'application $\mu:[u] \rightarrow\left(s^{+}\right)^{-1}\left([u] \cap \Omega_{\nu}\right)$, l'espace $[\dot{\mathbf{Q}}]$ est homéomorphe à l'espace des demi-droites affines de $\mathfrak{n}^{+}$dont la direction est dans $\mathfrak{z}^{+}$.

(ii) Il existe une section continue $s:[\dot{\mathbf{Q}}] \rightarrow \dot{\mathbf{Q}}$.

Preuve. - On commence par remarquer que, pour tout $b \in P$, on a la relation d'équivariance :

$$
\mu(b \cdot[u])=\rho(b) \cdot \mu([u]) .
$$

Ensuite, la formule de changement de cartes (2) assure que si $u \in \mathfrak{z}^{-}$alors $\mu([u])$ est la demi-droite $\left[a_{u} \cdot \frac{\theta u}{\|u\|^{2}}, \frac{\theta u}{\|u\|}\right)$. Par conséquent, pour tout $u \in \mathfrak{z}^{-}, \mu([u])$ est bien une demidroite. Maintenant, tout $u^{\prime} \in \mathbf{Q}$ s'écrit $A d(b) . u$ pour $b \in P$ et $u \in \mathfrak{z}^{-}$. De la relation 
$\mu(b .[u])=\rho(b) . \mu([u])$ et de la remarque 2 , on conclut que l'image de $\mu$ est contenue dans l'espace des demi-droites affines de $\mathfrak{n}^{+}$, dont la direction est dans $\mathfrak{z}^{+}$. Comme $\mu$ est clairement un homéomorphisme sur son image, il ne reste plus qu'à montrer que $\mu$ est surjective. Par la formule (2), il est clair que toute demi-droite de la forme $[v, v)$, avec $v \in S_{\mathfrak{z}^{+}}$, est atteinte par $\mu$. Puis en faisant agir $\rho\left(N^{+}\right)$sur ces demi-droites, on obtient que toutes les demi-droites de $\mathfrak{n}^{+}$, dont la direction est dans $\mathfrak{z}^{+}$, sont atteintes.

Il reste à définir la section $s$. On commence par définir $\tilde{s}: \mathfrak{n}^{+} \times S_{\mathfrak{z}^{+}} \rightarrow \mathbf{\mathbf { Q }} \operatorname{par} \tilde{s}:[x, v) \mapsto$ $A d\left(\operatorname{Exp}_{G}(x-v)\right) \cdot\left(a_{\theta v} \cdot \theta v\right)$. Puis on pose $s=\tilde{s} \circ \mu$, et on vérifie que $s$ est bien une section :

$$
\begin{aligned}
{[\tilde{s}([x, v))] } & =\operatorname{Exp}_{G}(x-v) \cdot\left[a_{\theta v} \cdot \theta v\right] \\
& =\mu^{-1}\left(\rho\left(\operatorname{Exp}_{G}(x-v)\right) \cdot[v, v)\right)=\mu^{-1}([x, v)) .
\end{aligned}
$$

LEMME 2. - L'espace $[\mathbf{Q}]$ vérifie les propriétés suivantes:

$\left(P_{1}\right)$ Pour tout point $x \in \mathbf{X}$, il existe un segment $\alpha \in[\mathbf{Q}]$ qui relie o et $x$.

$\left(P_{2}\right)$ Soit $\left(\alpha_{k}\right)$ une suite de $[\mathbf{Q}]$ qui tend vers $[o]$; alors $\lim _{k \rightarrow+\infty} L^{-}\left(\alpha_{k}\right)=0$, où $L^{-}\left(\alpha_{k}\right)$ représente la longueur du segment $\alpha_{k}$ pour la métrique $\rho^{-}$.

Preuve. - La propriété $\left(P_{1}\right)$ est triviale si $x=o$. On suppose donc $x \in \Omega_{\nu}$, et l'on considère $y=\left(s^{+}\right)^{-1}(x)$. Alors, pour tout $u \in S_{\mathfrak{z}^{+}}, \mu^{-1}([y, u))$ est un segment de $[\mathbf{Q}]$ qui relie $o$ et $x$.

Il reste à prouver $\left(P_{2}\right)$. Pour cela, on raisonne par l'absurde, et l'on suppose qu'il existe une suite de segments $\alpha_{k} \in[\dot{\mathbf{Q}}]$ qui tendent vers $[o]$, et tels que $L^{-}\left(\alpha_{k}\right)>\epsilon>0$, pour tout $k \in \mathbf{N}$. On identifie $[\dot{\mathbf{Q}}]$ avec les demi-droites de $\mathfrak{n}^{+}$dont la direction est dans $\mathfrak{z}^{+}$, si bien que l'on note $\alpha_{k}=\left[x_{k}, u_{k}\right)$, avec $x_{k} \in \mathfrak{n}^{+}$et $u_{k} \in S_{\mathfrak{z}^{+}}$. D'autre part, quitte à considérer une suite extraite, on supposera par la suite que $u_{k}$ a une limite $u_{\infty} \in S_{\mathfrak{z}^{+}}$. Nous utiliserons dans la démonstration le critère suivant, dont la preuve est aisée :

Lemme 3. - Soit $\left[x_{k}, u_{k}\right)$ une suite de $[\dot{\mathbf{Q}}]$. On suppose que $x_{k} \rightarrow \infty$ (i.e. $\left(x_{k}\right)$ sort de tout compact de $\left.\mathfrak{n}^{+}\right)$, et qu'il existe $u_{\infty}$ et $v_{\infty}$ dans $S_{\mathfrak{z}^{+}}$tels que $\lim _{k \rightarrow+\infty} u_{k}=u_{\infty}$ et $\lim _{k \rightarrow+\infty} \frac{x_{k}}{\left\|x_{k}\right\|}=v_{\infty}$. Alors la suite $\left[x_{k}, u_{k}\right)$ tend vers $[o]$ si et seulement si $v_{\infty} \neq-u_{\infty}$.

Soit $a^{t}$ le flot de Cartan introduit en section 2.1. L'action de $a^{t}$ sur $\mathfrak{n}^{-}$se fait par des transformations de la forme

$$
\left(\begin{array}{cc}
e^{-2 t} \operatorname{Id}_{\mathfrak{z}^{-}} & 0 \\
0 & e^{-t} \operatorname{Id}_{\mathfrak{n}_{1}^{-}}
\end{array}\right) .
$$

Ainsi, la norme associée à n'importe quel produit scalaire sur $\mathfrak{n}^{-}$est contractée exponentiellement par $A d\left(a^{t}\right)$ (lorsque $t \geqslant 0$ ). Il en résulte que $a^{t}$ agit également par contractions pour la métrique $\rho^{-}$: il existe une constante $c>0$ telle que $L^{-}\left(a^{t} .[\sigma]\right) \leqslant e^{-c t} L^{-}([\sigma])$.

D'autre part, toute suite de points $\left(x_{k}\right)$ qui part à l'infini dans $\mathfrak{n}^{+}$peut être ramenée dans un compact de $\mathfrak{n}^{+} \backslash\left\{0_{\mathfrak{n}^{+}}\right\}$par l'action d'une suite $A d\left(a^{t_{k}}\right)$, avec $\lim _{k \rightarrow+\infty} t_{k}=-\infty$. Autrement dit, il existe une suite $\left(t_{k}\right)$ avec $\lim _{k \rightarrow+\infty} t_{k}=-\infty$ telle que, quitte à considérer une suite extraite, $\sigma_{k}=a^{t_{k}} \cdot \alpha_{k}$ tend vers $\sigma_{\infty}=\left[x_{\infty}, u_{\infty}\right)$, avec $x_{\infty} \neq 0_{\mathfrak{n}^{+}}$.

Si l'on n'est pas dans le cas où $x_{\infty}=-\mu u_{\infty}$, pour un certain $\mu>0$, le segment $\sigma_{\infty}$ ne rencontre pas $0_{\mathfrak{n}^{+}}$, et par conséquent, $L^{-}\left(\left[\sigma_{k}\right]\right)$ est bornée supérieurement par $M$, pour tout $k \in \mathbf{N}$. Cela conduit à une majoration $L^{-}\left(\alpha_{k}\right) \leqslant M e^{c t_{k}}$, qui est incompatible avec l'hypothèse $L^{-}\left(\alpha_{k}\right)>\epsilon$, car $t_{k} \rightarrow-\infty$.

Maintenant, s'il existe $\mu>0$ tel que $x_{\infty}=-\mu u_{\infty}$. On écrit $x_{k}=y_{k}+z_{k}$, avec $y_{k} \in \mathfrak{n}_{1}^{+}$et $z_{k} \in$ $\mathfrak{z}^{+}$. On a $\lim _{k \rightarrow+\infty} e^{t_{k}} y_{k}=0$ et $\lim _{k \rightarrow+\infty} e^{2 t_{k}} z_{k}=x_{\infty}=-\mu u_{\infty}$. Ainsi, $\lim _{k \rightarrow+\infty} e^{2 t_{k}}\left(y_{k}+\right.$ $\left.z_{k}\right)=-\mu u_{\infty}$, ce qui implique $\lim _{k \rightarrow+\infty} \frac{x_{k}}{\left\|x_{k}\right\|}=-u_{\infty}$. Mais le lemme 3 assure alors que la suite $\left[x_{k}, u_{k}\right)$ ne tendait pas vers $[o]$, une contradiction.

$4^{\mathrm{e}}$ SÉRIE - TOME $40-2007-\mathrm{N}^{\circ} 5$ 


\subsection{Aspects dynamiques}

\subsubsection{Quelques définitions générales}

On commence par introduire quelques notions dynamiques que nous utiliserons tout au long de l'article.

Soient $M$ une variété et $G$ un sous-groupe d'homéomorphismes de $M$.

DÉFINITION 2 (Donnée stable).-On appelle donnée stable de $G$ un quadruplet $\left(f_{k}, x_{k}\right.$, $\left.x_{\infty}, y_{\infty}\right)$, où $\left(f_{k}\right)$ est une suite de $G,\left(x_{k}\right)$ est une suite de $M$ qui converge vers le point $x_{\infty} \in M$, et telle que la suite $y_{k}=f_{k}\left(x_{k}\right)$ converge vers le point $y_{\infty} \in M$.

Il est facile de vérifier qu'un sous-groupe $G$ de $\operatorname{Homeo}(M)$, le groupe des homéomorphismes de $M$, n'agit pas proprement (i.e. possède une suite qui n'agit pas proprement) si et seulement si il existe une donnée stable $\left(f_{k}, x_{k}, x_{\infty}, y_{\infty}\right)$, où $\left(f_{k}\right)$ est une suite de $G$ tendant vers l'infini dans $H_{o m e o}(M)$ (on dit qu'une suite tend vers l'infini dans $\operatorname{Homeo}(M)$ si son intersection avec tout compact de Homeo( $M)$ est finie).

DÉFInITION 3 (Équicontinuité). - Soit $\left(f_{k}, x_{k}, x_{\infty}, y_{\infty}\right)$ une donnée stable de $G$. On dit que l'action de $\left(f_{k}\right)$ est équicontinue en $x_{\infty}$ s'il existe une suite extraite $\left(f_{n_{k}}\right)$ de $\left(f_{k}\right)$, telle que, pour toute suite $x_{k}^{\prime}$ qui tend vers $x_{\infty}, f_{n_{k}}\left(x_{k}^{\prime}\right)$ tend vers $y_{\infty}$.

\subsubsection{Dynamique Nord-Sud sur $X$}

LEMME 4. - Soit $\left(g_{k}\right)$ une suite de $G$ qui tend vers l'infini. Alors quitte à considérer une sous-suite de $\left(g_{k}\right)$, il existe deux points $o^{+}$et $o^{-}$de $\mathbf{X}$ (éventuellement confondus), tels que $\left(g_{k}\right)$ ait les propriétés dynamiques suivantes:

(i) Pour tout compact $K$ de $\Omega_{o^{+}}=\mathbf{X} \backslash\left\{o^{-}\right\}, \lim _{k \rightarrow+\infty} g_{k}(K)=o^{+}$.

(ii) Pour tout compact $K$ de $\Omega_{0^{-}}=\mathbf{X} \backslash\left\{o^{+}\right\}, \lim _{k \rightarrow+\infty}\left(g_{k}\right)^{-1}(K)=o^{-}$.

Preuve. - Le groupe $G$ admet la décomposition de Cartan $G=K A K, K$ désignant le compact maximal de $G$. Ainsi, il suffit de démontrer le lemme pour $g_{k}=a_{k}$, une suite du groupe $A$ qui tend vers l'infini. Quitte à changer $a_{k}$ en $a_{k}^{-1}$, ce qui reviendra à permuter $o^{+}$et $o^{-}$, on obtient que $a_{k}=a^{t_{k}}$, avec $t_{k} \rightarrow+\infty$. Au vu de l'expression matricielle pour l'action de $A d\left(a^{t}\right)$ sur $\mathfrak{n}^{-}$, donnée dans la preuve du lemme 2 , il est clair que, pour tout compact $\mathcal{K}$ de $\mathfrak{n}^{-}, \operatorname{Ad}\left(a^{t}\right) . \mathcal{K}$ tend uniformément vers 0 lorsque $t \rightarrow+\infty$. Maintenant, de la relation $s^{-}\left(A d\left(a^{t_{k}}\right) \cdot u\right)=a^{t_{k}} \cdot s^{-}(u)$, on déduit le point (i) du lemme, en posant $o^{+}=s^{-}(0)$. Le point (ii) s'obtient en appliquant la même analyse à $a^{-t_{k}}$ sur $\mathfrak{n}^{+}$, et en posant $o^{-}=s^{+}(0)$.

Par analogie avec ce qui se passe pour la sphère conforme standard, nous appellerons $o^{+}$et $o^{-}$les pôles de $\left(g_{k}\right)$.

Une conséquence du lemme 4 est le :

Corollaire 1. - Soit $\left(b_{k}\right)$ une suite de P qui part à l'infini, de pôles $o^{+}$et $o^{-}$. L'action de $\left(b_{k}\right)$ est équicontinue en o si et seulement si $o=o^{+}$, et $o^{-} \neq 0$. Dans ce cas, il existe $l_{1, k}$ et $l_{2, k}$ deux suites de $P$ relativement compactes dans $P$ telles que $b_{k}=l_{1, k} a_{k} l_{2, k}$, où $a_{k} \in A$.

Preuve. - Si $\left(b_{k}\right)$ est une suite de $P$ qui tend vers l'infini, l'analyse dynamique faite dans le lemme montre d'une part que les seuls points fixes possibles pour $\left(b_{k}\right)$ sont $o^{+}$et $o^{-}$, et d'autre part que l'action de $\left(b_{k}\right)$ n'est pas équicontinue en $o^{-}$(elle l'est en revanche sur $\Omega_{o^{+}}$). Donc $o=o^{+}$, et $o^{-} \neq o^{+}$. Maintenant, toujours par le lemme précédent, $\lim _{k \rightarrow+\infty} b_{k}^{-1} \cdot \nu=o^{-}$. Comme $o^{-} \in \Omega_{\nu}$, il existe une suite $n_{k}$ de $N^{+}$, relativement compacte dans $N^{+}$, telle que $n_{k} b_{k}^{-1} . \nu=\nu$. Mais du coup, $n_{k} b_{k}^{-1}$ fixe $o$ et $\nu$, donc est dans $L=A M$. Ainsi, il existe $m_{k}$ une suite de $M$ telle que $n_{k} b_{k}^{-1} m_{k}$ soit dans $A$, ce qui achève la preuve du corollaire. 


\subsubsection{Dynamique de $P$ sur $[\mathbf{Q}]$}

Proposition 2. - Soit $\left(b_{k}\right)$ une suite de $P$ qui tend vers l'infini. Alors, quitte à considérer éventuellement $\left(b_{k}^{-1}\right)$ à la place de $\left(b_{k}\right)$, et à prendre une suite extraite :

(i) Il existe un ouvert $\boldsymbol{\Omega}^{+} \subset[\dot{\mathbf{Q}}]$, contenant $[o]$ dans son adhérence, tel que si $K$ est un compact de $\boldsymbol{\Omega}^{+}, b_{k}$.K tend vers $[o]$ lorsque $k$ tend vers $+\infty$.

(ii) L'ensemble $s\left(\boldsymbol{\Omega}^{+}\right)$contient dans son adhérence des éléments de $\mathfrak{z}^{-} \backslash\left\{0_{\mathfrak{g}}\right\}$ arbitrairement proches de $0_{\mathfrak{g}}$.

Preuve. - Le point (ii) est un point technique qui nous sera utile dans la section 6.

Dans toute la preuve, on identifie $[\dot{\mathbf{Q}}] \mathbf{a ̀ ~}^{+} \times S_{\mathfrak{z}^{+}}$via l'application $\mu$. Les conclusions $d u$ lemme 2 ne sont pas altérées si l'on compose $\left(b_{k}\right)$ par une suite du sous-groupe compact $M \subset P$ (en effet, $A d(M)$ préserve $\mathfrak{z}^{-}$). On supposera donc dans toute la preuve que $\left(b_{k}\right)$ est une suite de $A N^{+} \subset P$, et nous écrirons $b_{k}=a^{t_{k}} n_{k}^{+}$. Sur $\mathfrak{n}^{+}, \rho\left(b_{k}\right)=L_{k}+T_{k}$, c'est-à-dire que $\rho\left(b_{k}\right)$ est la composée de l'application linéaire $L_{k}$ et de la translation de vecteur $T_{k}$. Dans une base compatible avec la graduation $\mathfrak{n}^{+}=\mathfrak{n}_{1}^{+} \oplus \mathfrak{z}^{+}$, la matrice de $L_{k}$ est de la forme $\left(\begin{array}{cc}e^{t_{k}} & 0 \\ D_{k} & e^{2 t_{k}}\end{array}\right)$.

Quitte à considérer une suite extraite de $\left(b_{k}\right)$, et quitte à remplacer $\left(b_{k}\right)$ par $\left(b_{k}^{-1}\right)$, nous supposerons que $t_{k}$ admet une limite dans $\mathbf{R}_{+}^{*} \cup\{+\infty\}$. Ainsi, en considérant à nouveau une suite extraite, il existe deux suites de $\mathbb{R}^{+}, \beta_{k}$ et $\mu_{k}$, qui tendent vers $\beta_{\infty}$ et $\mu_{\infty}$ dans $\mathbb{R}_{+}^{*} \cup\{+\infty\}$ et $\mathbb{R}^{+} \cup\{+\infty\}$ respectivement, et une suite $B_{k}$ (resp. $\tau_{k}$ ) de $\operatorname{End}\left(\mathfrak{n}^{+}\right)$(resp. de $S_{\mathfrak{n}^{+}}$) qui converge vers $B_{\infty} \neq 0$ dans $\operatorname{End}\left(\mathfrak{n}^{+}\right)$(resp. $\left.\tau_{\infty} \in S_{\mathfrak{n}^{+}}\right)$, de telle sorte que $\rho\left(b_{k}\right)=\beta_{k} B_{k}+\mu_{k} \tau_{k}$, et que l'on soit dans un des trois cas suivants :

- Premier cas : $\lim _{k \rightarrow+\infty} \frac{\beta_{k}}{\mu_{k}}=0$.

Soit

$$
F=\left\{[x, u) \in[\dot{\mathbf{Q}}] \mid u=-\tau_{\infty}\right\} .
$$

C'est un fermé dans $[\dot{\mathbf{Q}}]$ (éventuellement vide si $-\tau_{\infty} \notin S_{\mathfrak{z}^{+}}$). Soit $\left[x_{k}, u_{k}\right.$ ) une suite de $\boldsymbol{\Omega}^{+}=[\dot{\mathbf{Q}}] \backslash F$ qui converge vers $\left[x_{\infty}, u_{\infty}\right) \in \boldsymbol{\Omega}^{+}$. On a $\rho\left(b_{k}\right)\left[x_{k}, u_{k}\right)=\left[x_{k}^{\prime}, u_{k}\right)$, avec $x_{k}^{\prime}=$ $\mu_{k}\left(\frac{\beta_{k}}{\mu_{k}} B_{k} \cdot x_{k}+\tau_{k}\right)$. Sous nos hypothèses, $\mu_{k} \rightarrow+\infty$ et donc $\lim _{k \rightarrow+\infty} \frac{x_{k}^{\prime}}{\left\|x_{k}^{\prime}\right\|}=\tau_{\infty}$. Comme $u_{\infty} \neq-\tau_{\infty}$, le lemme 3 s'applique et on obtient bien que $\lim _{k \rightarrow+\infty} \rho\left(b_{k}\right)\left[x_{k}, u_{k}\right)=[o]$.

Si $x \in \mathfrak{z}^{-}$, on constate que $\left[\theta x, \frac{\theta x}{\|x\|}\right)$ et $\left[-\theta x, \frac{-\theta x}{\|x\|}\right)$ ne peuvent pas être dans $F$ simultanément. Cela prouve que $s\left(\boldsymbol{\Omega}^{+}\right)$contient des éléments de $\mathfrak{z}^{-}$arbitrairement proches de $0_{\mathfrak{g}}$. Cela prouve le point (ii) dans ce cas.

- Second cas $: \lim _{k \rightarrow+\infty} \frac{\beta_{k}}{\mu_{k}}=+\infty$.

Posons :

$$
F=\left\{[x, u) \in[\dot{\mathbf{Q}}] \mid B_{\infty} . x \in \mathbb{R}^{-} . u\right\} .
$$

Il s'agit d'un fermé de $[\dot{\mathbf{Q}}]$. Remarquons que, dans ce cas-ci, on a forcément $\beta_{\infty}=+\infty$. Dans le cas contraire, on devrait avoir $\mu_{\infty}=0$, et $\beta_{\infty} \in \mathbb{R}_{+}^{*}$. Dans ce cas, $L_{k}$ convergerait vers $L_{\infty} \in G L\left(\mathfrak{n}^{+}\right)$, et la suite $\left(b_{k}\right)$ serait bornée.

Soit $\left[x_{k}, u_{k}\right)$ une suite de $\boldsymbol{\Omega}^{+}=[\mathbf{Q}] \backslash F$ qui converge vers $\left[x_{\infty}, u_{\infty}\right) \in \mathbf{\Omega}^{+}$. On a $\rho\left(b_{k}\right)\left[x_{k}, u_{k}\right)=\left[x_{k}^{\prime}, u_{k}\right)$, avec $x_{k}^{\prime}=\beta_{k}\left(B_{k} \cdot x+\frac{\mu_{k}}{\beta_{k}} \tau_{k}\right)$, et $\lim _{k \rightarrow+\infty} \frac{x_{k}^{\prime}}{\left\|x_{k}^{\prime}\right\|}=\frac{B_{\infty} \cdot x_{\infty}}{\left\|B_{\infty} \cdot x_{\infty}\right\|}$. Par définition de $F, B_{\infty} x_{\infty} \notin \mathbb{R}^{-} . u_{\infty}$. Le lemme 3 s'applique, et on obtient $\lim _{k \rightarrow+\infty} \rho\left(b_{k}\right)\left[x_{k}, u_{k}\right)=$ $[o]$.

Comme on l'a déjà noté, $\beta_{\infty}=+\infty$. Dans une base qui respecte la graduation $\mathfrak{n}^{+}=\mathfrak{n}_{1}^{+} \oplus \mathfrak{z}^{+}$, la matrice $B_{\infty}$ est soit de la forme $\left(\begin{array}{cc}0 & 0 \\ A_{\infty} & 0\end{array}\right)$, avec $A_{\infty} \neq 0$ (cette éventualité ne peut advenir que lorsque $\mathfrak{g} \neq \mathfrak{s o}(1, n), \mathfrak{s u}(1,1)$ ou $\mathfrak{s p}(1,1))$, soit de la forme $\left(\begin{array}{cc}0 & 0 \\ C_{\infty} & \operatorname{Id}_{\mathfrak{z}^{+}}\end{array}\right)$, avec éventuellement $C_{\infty}=0$.

$4^{\text {e }}$ SÉRIE - TOME $40-2007-\mathrm{N}^{\circ} 5$ 
Dans le second cas, si $x \in \mathfrak{z}^{-} \backslash\left\{0_{\mathfrak{g}}\right\}$, alors $B_{\infty} \cdot \theta x=\theta x$, et donc $B_{\infty} \cdot \theta x \notin \mathbb{R}^{-} \cdot \frac{\theta x}{\|x\|}$. Par conséquent $\mathfrak{z}^{-} \backslash\left\{0_{\mathfrak{g}}\right\} \subset s\left(\boldsymbol{\Omega}^{-}\right)$, et le point (ii) est démontré. Dans le premier cas, on choisit une suite $y_{n}$ de $\mathfrak{n}^{+}$qui tend vers $0_{\mathfrak{g}}$ et telle que, pour tout $n \in \mathbf{N}, A_{\infty} \cdot y_{n} \notin \mathbb{R}^{-} \cdot \frac{\theta x}{\|x\|}$. Alors pour $x \in \mathfrak{z}^{-} \backslash\left\{0_{\mathfrak{g}}\right\}, s\left(\left[\theta x+y_{n}, \frac{\theta x}{\|x\|}\right)\right)$ est une suite de $s\left(\boldsymbol{\Omega}^{+}\right)$qui tend vers $x$, ce qui montre le point (ii).

- Troisième cas : $\lim _{k \rightarrow+\infty} \frac{\beta_{k}}{\mu_{k}}=\alpha$, avec $\alpha \in \mathbb{R}_{+}^{*}$.

On remarque, comme dans le cas précédent, que comme $\left(b_{k}\right)$ tend vers l'infini, on a nécessairement $\beta_{\infty}=\mu_{\infty}=+\infty$.

Posons :

$$
F=\left\{[x, u) \in[\dot{\mathbf{Q}}] \mid \alpha B_{\infty} . x+\tau_{\infty} \in \mathbb{R}^{-} . u\right\} .
$$

Il s'agit d'un fermé de $[\dot{\mathbf{Q}}]$. Soit $\left[x_{k}, u_{k}\right)$ une suite de $\boldsymbol{\Omega}^{+}=[\dot{\mathbf{Q}}] \backslash F$ qui converge vers $\left[x_{\infty}, u_{\infty}\right) \in \boldsymbol{\Omega}^{+}$. On a $\rho\left(b_{k}\right)\left[x_{k}, u_{k}\right)=\left[x_{k}^{\prime}, u_{k}\right)$, et $x_{k}^{\prime}=\mu_{k}\left(\frac{\beta_{k}}{\mu_{k}} B_{k} \cdot x_{k}+\tau_{k}\right)$. Comme $\left[x_{\infty}, u_{\infty}\right) \in[\dot{\mathbf{Q}}] \backslash F$, on a $\alpha B_{\infty} . x_{\infty}+\tau_{\infty} \neq 0$, donc $\lim _{k \rightarrow+\infty} \frac{x_{k}^{\prime}}{\left\|x_{k}^{\prime}\right\|}=\frac{\alpha B_{\infty} \cdot x_{\infty}+\tau_{\infty}}{\left\|\alpha B_{\infty} \cdot x_{\infty}+\tau_{\infty}\right\|}$. Comme $\alpha B_{\infty} . x_{\infty}+\tau_{\infty} \notin \mathbb{R}^{-} . u_{\infty}$, on applique une nouvelle fois le lemme 3, et on conclut : $\lim _{k \rightarrow+\infty} \rho\left(b_{k}\right)\left[x_{k}, u_{k}\right)=[o]$.

Comme on est encore dans le cas $\beta_{\infty}=+\infty$, les expressions matricielles possibles de $B_{\infty}$ sont les mêmes que dans le cas précédent. On vérifie alors que, lorsque $x \in \mathfrak{z}^{-}$est très proche de $0_{\mathfrak{g}},\left[\theta x, \frac{\theta x}{\|x\|}\right)$ et $\left[-\theta x,-\frac{\theta x}{\|x\|}\right)$ ne peuvent être simultanément dans $F$, ce qui prouve le point (ii).

\section{Géométries de Cartan}

Nous n'introduisons ici que le matériel de base sur les géométries de Cartan. Pour des détails sur le sujet, et les preuves de certaines propositions et lemmes ci-dessous, nous renvoyons à l'excellente référence [24]. On considère dans toute cette section une géométrie de Cartan $(M, B, \omega)$, modelée sur $\mathbf{X}=G / P$ (en fait, pour les généralités qui suivent, $G$ est un groupe de Lie quelconque, et $P$ un sous-groupe fermé de $G$ ).

\subsection{Parallélisme et métrique riemannienne sur $B$}

Soit $\left(E_{1}, \ldots, E_{s}\right)$ une base de l'algèbre de Lie $\mathfrak{g}$, orthonormée pour la métrique \langle\rangle$_{\mathfrak{g}}$ introduite en section 2.2. On définit alors en chaque point $p$ de $B$ un repère $\mathcal{R}(p)=\left(E_{1}^{\dagger}(p), \ldots, E_{s}^{\dagger}(p)\right)$ par $E_{k}^{\dagger}(p)=\omega_{p}^{-1}\left(E_{k}\right)$. Ce champ de repères fournit un parallélisme $\mathcal{R}$ sur $B$. Pour tout vecteur $\xi \in \mathfrak{g}$, s'écrivant $\xi=\Sigma \lambda_{k} E_{k}$, on peut définir un champ de vecteurs $\xi^{\dagger}$ sur $B$ par $\xi^{\dagger}(p)=\Sigma \lambda_{k} E_{k}^{\dagger}(p)$. De tels champs, ayant des coordonnées constantes dans $\mathcal{R}$ seront qualifiés de parallèles.

Notation 1. - On adoptera souvent la notation $\xi_{p}$ à la place de $\xi^{\dagger}(p)$. De même, si $\boldsymbol{\Lambda}$ est un sous-ensemble de $\mathfrak{g}$, on peut lui associer un champ de "sous-ensembles parallèles" dans $T B$, définis par $\Lambda_{p}=\omega_{p}^{-1}(\boldsymbol{\Lambda})$, pour tout $p \in B$. Dans le cas d'une suite $\boldsymbol{\Lambda}_{k}$ de sous-ensembles, on notera $\Lambda_{p, k}=\omega_{p}^{-1}\left(\boldsymbol{\Lambda}_{k}\right)$.

Pour une sous-algèbre de Lie $\mathfrak{h} \subset \mathfrak{g}$, on notera $H_{p, \mathfrak{h}}=\omega_{p}^{-1}(\mathfrak{h})$.

DÉFINITION 4. - On appelle $\rho$ la métrique riemannienne sur $B$ qui fait de $\mathcal{R}(p)$ un repère orthonormé pour tout $p \in B$.

Remarquons que pour tout $p \in B, \rho_{p}=\omega_{p}^{*}\left(\langle\rangle_{\mathfrak{g}}\right)$. 


\subsection{Courbure}

La forme de Maurer-Cartan sur un groupe de Lie $G$ satisfait l'équation dite de structure : $d \omega_{G}+\frac{1}{2}\left[\omega_{G}, \omega_{G}\right]=0$. Bien entendu, pour une connexion de Cartan $\omega$ quelconque, la 2-forme $\Omega=d \omega+\frac{1}{2}[\omega, \omega]$ n'est généralement pas triviale. On appelle $\Omega$ la courbure de la connexion $\omega$. Voici quelques propriétés de la courbure :

(i) Pour tout $b \in P,\left(R_{b}\right)^{*} \Omega=\operatorname{Ad}\left(b^{-1}\right) \Omega$.

(ii) $\Omega(X, Y)=0$ dès que $X$ ou $Y$ est un vecteur vertical (i.e. tangent aux fibres de $B \rightarrow M$ ). Une géométrie de Cartan $(M, B, \omega)$ est dite plate si $\Omega$ est nulle sur $B$.

Remarquons que, par la propriété d'équivariance $(i)$, il suffit que la courbure s'annule en un point d'une fibre pour qu'elle s'annule sur la fibre toute entière. Lorsque $x$ est un point de $M$, on dira, par abus de langage, que $\Omega$ s'annule en $x$, pour signifier que $\Omega$ est nulle sur toute la fibre au-dessus de $x$.

Exemple 1. - Si $U$ est un ouvert de $\mathbf{X}$ stable par un sous-groupe discret $\Gamma \subset G$, et si l'action de $\Gamma$ sur $U$ est propre, discontinue sans point fixe, alors la variété $M=\Gamma \backslash U$ est naturellement munie d'une géométrie de Cartan plate, héritée de $\mathbf{X}$. Le fibré $B$ est alors le quotient $\Gamma \backslash G$ et la connexion de Cartan est $\bar{\omega}_{G}$, la forme induite par $\omega_{G}$ sur $\Gamma \backslash G$.

\subsubsection{Connexions régulières}

Disons à présent quelques mots sur l'hypothèse faite sur la connexion $\omega$, dans l'énoncé du théorème 1 . On suppose que $(M, B, \omega)$ est modelé sur l'espace $\mathbf{X}=G / P$, tel que l'algèbre de Lie $\mathfrak{g}$ est $k$-graduée. Cela signifie que $\mathfrak{g}=\mathfrak{g}_{-k} \oplus \cdots \oplus \mathfrak{g}_{0} \oplus \cdots \oplus \mathfrak{g}_{k}, k \in \mathbf{N}^{*}$, et que l'algèbre de Lie de $P$ est $\mathfrak{p}=\mathfrak{g}_{0} \oplus \cdots \oplus \mathfrak{g}_{k}$. De plus, pour tout $-k \leqslant i \leqslant j \leqslant k,\left[\mathfrak{g}_{i}, \mathfrak{g}_{j}\right] \subset \mathfrak{g}_{i+j}$. On obtient alors une filtration $A d(P)$-invariante $\mathfrak{g}^{-k}=\mathfrak{g} \supset \mathfrak{g}^{-k+1} \supset \cdots \supset \mathfrak{g}^{k}=\mathfrak{g}_{k}$, en posant $\mathfrak{g}^{i}=\mathfrak{g}_{i} \oplus \mathfrak{g}_{i+1} \oplus \cdots \oplus \mathfrak{g}_{k}$.

DÉFInItion 5 (Régularité). - On dit que la connexion de Cartan $\omega$ est régulière si, pour tout $\xi \in \mathfrak{g}^{i}$ et $\zeta \in \mathfrak{g}^{j}, i, j<0$, et pour tout $p \in B, \Omega_{p}\left(\xi_{p}, \zeta_{p}\right) \in \mathfrak{g}^{i+j+1}$.

Cette notion de régularité est importante, car dans la plupart des cas où l'on sait résoudre le problème d'équivalence la connexion de Cartan canonique obtenue est régulière (la régularité étant une des conditions de normalisation sur la connexion qui assurent son unicité).

Regardons plus précisément ce que signifie la régularité dans le cas où $\mathfrak{g}$ est une algèbre de Lie simple de rang 1.

- Si les racines de $\mathfrak{g}$ sont $\{-\alpha, \alpha\}$, alors la condition de régularité n'impose aucune condition sur la courbure $\Omega$.

- Si les racines de $\mathfrak{g}$ sont $\{-2 \alpha,-\alpha, \alpha, 2 \alpha\}$, alors la condition de régularité impose que, pour tout couple $(\xi, \zeta)$ dans $\mathfrak{g}^{-1}=\mathfrak{n}_{1}^{-} \oplus \mathfrak{p}, \Omega_{p}\left(\xi_{p}, \zeta_{p}\right)$ appartienne à $\mathfrak{g}^{-1}$, pour tout $p \in B$.

\subsection{Développement des courbes}

Une des propriétés fondamentales d'une connexion de Cartan est qu'elle permet d'établir un lien entre les courbes paramétrées de la variété $M$ passant par un point donné, et les courbes de l'espace modèle $\mathbf{X}$.

DÉfinition 6.- Soient $N$ une variété, $q$ un point de $N$ et $I \subset \mathbb{R}$ un intervalle ouvert contenant 0 . On note $C^{1}(N, I, q)$ l'espace des courbes $\gamma: I \rightarrow N$, de classe $C^{1}$ et telles que $\gamma(0)=q$.

Commençons par rappeler le lemme de développement des courbes pour une géométrie de Cartan (voir [24], lemme 4.12 p. 208 pour une preuve) :

$4^{\mathrm{e}}$ SÉRIE - TOME $40-2007-\mathrm{N}^{\circ} 5$ 
LEMME 5. - Soient p un point de B, et I un intervalle ouvert contenant 0. Alors il existe une unique application $\left.\hat{\mathcal{D}}_{p}: C^{1}(B, I, p) \rightarrow C^{1}(G, I, e)\right)$ qui vérifie :

(i) pour toute courbe $\hat{\gamma}$ dans $C^{1}(B, I, p)$, la courbe $\alpha=\hat{\mathcal{D}}_{p}(\hat{\gamma})$ satisfait pour tout $t \in I$ : $\omega\left(\hat{\gamma}^{\prime}(t)\right)=\omega_{G}\left(\alpha^{\prime}(t)\right)$.

(ii) si a $\in C^{1}\left(P, I, a_{0}\right)$, et si l'on note $R_{a} \hat{\gamma}$ la courbe définie par $\left(R_{a} \hat{\gamma}\right)(t)=R_{a(t)} \cdot \hat{\gamma}(t)$, alors $\hat{\mathcal{D}}_{p . a_{0}}\left(R_{a} \hat{\gamma}\right)=a_{0}^{-1} \cdot\left(R_{a} \alpha\right)$.

Corollaire 2. - Soient $\gamma \in C^{1}(M, I, x)$ et $\hat{\gamma}_{1}: I \rightarrow B, \hat{\gamma}_{2}: I \rightarrow B$ deux remontés de $\gamma$ dans $C^{1}(B, I, p)$. Alors $\hat{\mathcal{D}}_{p}\left(\hat{\gamma}_{1}\right)$ et $\hat{\mathcal{D}}_{p}\left(\hat{\gamma}_{2}\right)$ se projettent sur la même courbe de $\mathbf{X}$.

DÉFINITION 7 (Application développante). - Le corollaire affirme qu'étant donnés $\Delta x \in M$, et $\hat{x} \in B$ au-dessus de $x$, il existe une application bien définie de $C^{1}(M, I, x)$ dans $C^{1}(\mathbf{X}, I, o)$. On note cette application $\mathcal{D}_{x}^{\hat{x}}$ et on l'appelle application développante en $x . \operatorname{Si} \gamma \in C^{1}(M, I, x)$ et si $[\gamma]$ est le segment géométrique associé, on notera $\mathcal{D}_{x}^{\hat{x}}([\gamma])=\left[\mathcal{D}_{x}^{\hat{x}}(\gamma)\right]$.

\subsubsection{Développement pour les structures plates}

Nous renvoyons encore à [24] pour les détails concernant cette section.

L'annulation de la courbure est la seule obstruction pour que $(B, \omega)$ soit localement isomorphe à $\left(G, \omega_{G}\right)$.

On note $\tilde{M}$ le revêtement universel de $M$, et $r: \tilde{M} \rightarrow M$ l'application de revêtement. Il existe un revêtement $\tilde{r}: \tilde{B} \rightarrow B$, qui est un $P$-fibré principal $\tilde{\pi}: \tilde{B} \rightarrow \tilde{M}$, de sorte que le diagramme suivant commute :

$$
\begin{array}{ccc}
\tilde{B} & \stackrel{\tilde{r}}{\rightarrow} & B \\
\downarrow & & \\
\tilde{M} & \stackrel{r}{\rightarrow} & M
\end{array}
$$

D'autre part, $\tilde{r}^{*} \omega$ est une connexion de Cartan sur $\tilde{B}$.

THÉORÈME 2. - Si la courbure de $\omega$ est nulle, alors il existe un morphisme de fibrés $\tilde{\delta}:(\tilde{B}, \omega) \rightarrow\left(G, \omega_{G}\right)$, qui est une immersion satisfaisant $\tilde{\delta}^{*} \omega_{G}=\omega$. L'application $\tilde{\delta}$ induit une immersion $\delta: \tilde{M} \rightarrow \mathbf{X}$.

Dans ce cas, on dit que la variété $M$ est munie d'une $(G, \mathbf{X})$-structure, et l'application $\delta$ s'appelle une application développante de la structure. Soient $(M, B, \omega)$ une géométrie de Cartan plate, $x$ un point de $M$ et $\tilde{x}$ un point de $\tilde{M}$ au-dessus de $x$. Quitte à composer $\tilde{\delta}$ par un élément de $G$ à l'arrivée, on peut supposer que $\delta(\tilde{x})=o$. Si $\gamma \in C^{1}(M, I, x)$, alors il existe une unique $\tilde{\gamma} \in C^{1}(\tilde{M}, I, \tilde{x})$ telle que $r(\tilde{\gamma}(t))=\gamma(t)$ pour tout $t \in I$. Si $\hat{x}$ est le point au-dessus de $\tilde{x}$ de sorte que $\tilde{\delta}(\hat{x})=e$, alors $\mathcal{D}_{\tilde{x}}^{\hat{x}}(\tilde{\gamma})$ n'est autre que $\delta \circ \tilde{\gamma}$.

\section{Les géodésiques d'une géométrie parabolique de rang 1}

\subsection{Applications exponentielles}

Soient $p \in B$ et $\xi \in T_{p} B$; on note $U=\omega_{p}(\xi)$, et $\phi_{\xi}^{t}$ le flot local associé au champ parallèle $U^{\dagger}$. Il existe alors un voisinage $W_{p}^{B}$ de $0_{p}$ dans $T_{p} B$, étoilé autour de $0_{p}$, tel que, pour tout $\xi \in W_{p}^{B}$, $t \mapsto \phi_{\xi}^{t}(p)$ est défini sur $[0,1]$.

Notation 2. - Nous noterons $W^{B}$ l'ouvert de $T B$ défini par $\bigcup_{p \in B} W_{p}^{B}$. Si $p \in B, \mathbf{W}_{p}$ désignera le sous-ensemble de $\mathfrak{g}$ défini par $\omega_{p}\left(W_{p}^{B}\right)$. 
DÉfINITION 8. - On définit l'application exponentielle $\operatorname{Exp}: W^{B} \rightarrow B$, par $\operatorname{Exp}_{p}(\xi)=$ $\phi_{\xi}^{1}(p)$, pour tout $p \in B, \xi \in W_{p}^{B}$.

- Si $x=\pi(p)$ et $\xi \in W_{p}^{B}$, on note $\operatorname{Exp}_{x}(\xi)=\pi \circ \operatorname{Exp}_{p}(\xi)$.

- Pour $\xi \in W_{p}^{B}$, on appelle $\hat{\xi}^{*}$ (resp. $\xi^{*}$ ) la courbe de [0,1] dans $B$ (resp. de $[0,1]$ dans $M$ ) définie par $\hat{\xi}^{*}(t)=\operatorname{Exp}_{p}(t \xi)$ (resp. $\left.\xi^{*}(t)=\operatorname{Exp}_{x}(t \xi)\right)$. Le support géométrique de la courbe $\hat{\xi}^{*}$ (resp. $\left.\xi^{*}\right)$ sera noté $[\hat{\xi}]$ (resp. $[\xi]$ ).

- On notera $[x]$ à la place de $\left[0_{p}\right]$.

- Si $\Lambda_{p} \subset W_{p}^{B}$, on note $\left[\Lambda_{p}\right]=\bigcup_{\xi \in \Lambda_{p}}[\xi]$.

On remarque que $\operatorname{Exp}_{\pi(p)}\left(\Lambda_{p}\right) \subset\left[\Lambda_{p}\right]$.

\subsubsection{Géodésiques}

Nous supposons à présent que $(M, B, \omega)$ est une géométrie de Cartan modelée sur $\mathbf{X}=\partial \mathbf{H}_{\mathbb{K}}^{d}$.

DÉfinition 9 (Géodésiques sur $M$ ). - Soient $I$ un intervalle contenant $0, x$ un point de $M$, $\hat{x} \in B$ au-dessus de $x$, et $\gamma \in C^{1}(M, I, x)$. On dit que $\gamma$ est une géodésique paramétrée de $M$ si et seulement si $\mathcal{D}_{x}^{\hat{x}}(\gamma)$ est une géodésique paramétrée de $\mathbf{X}$, telle que celles-ci ont été définies en partie 2. Cette définition est indépendante du choix de $\hat{x}$ au-dessus de $x$, par $A d(P)$-invariance de $\mathbf{Q}$.

DÉfInItion 10. - Pour tout $p \in B$, on définit $Q_{p}=\left\{\xi \in T_{p} B \mid \omega_{p}(\xi) \in \mathbf{Q}\right\}$, et $\dot{Q}_{p}=$ $Q_{p} \backslash\left\{0_{p}\right\}$.

- $T Q=B \times{ }_{P} \mathbf{Q}$ est le sous-fibré de $T B$ dont les fibres sont les $Q_{p}$.

- Pour tout $p \in B$, on note $Q_{p}^{\text {reg }}$ la partie régulière de $Q_{p}$, c'est-à-dire l'ensemble des $u \in \dot{Q}_{p} \cap W_{p}^{B}$ tels que, si $x=\pi(p)$, la restriction de $\operatorname{Exp}_{x}$ à $Q_{p}$ soit une submersion en $u$.

Lemme 6.-Soient $x \in M$ et $\hat{x} \in B$ au-dessus de $x$. Alors les segments géodésiques paramétrés par $[0,1]$, valant $x$ en 0 sont exactement les $\xi^{*}$ pour $\xi \in W_{\hat{x}}^{B} \cap Q_{\hat{x}}$.

Preuve. - La définition même des champs parallèles conduit à la relation :

$$
\mathcal{D}_{x}^{\hat{x}}\left(\xi^{*}\right)=\left(\omega_{\hat{x}}(\xi)\right)^{*}
$$

Le lemme en découle immédiatement.

On énonce à présent un lemme très important pour la suite. Il fait le lien entre le comportement d'une suite de segments géodésiques sur $M$, et le comportement de leurs développements.

LEMME 7. - Soient $\hat{x}_{k}$ une suite de $B$ qui converge vers $\hat{x}_{\infty} \in B$ et $\boldsymbol{\Lambda}_{k}$ une suite de parties de $\mathbf{Q} \cap \mathbf{W}_{\hat{x}_{k}}$. Alors si $\lim _{k \rightarrow+\infty}\left[\boldsymbol{\Lambda}_{k}\right]=[o]$, on a $\lim _{k \rightarrow+\infty}\left[\Lambda_{\hat{x}_{k}, k}\right]=\left[x_{\infty}\right]$.

Preuve. - On a, comme indiqué plus haut, adopté la notation $\Lambda_{\hat{x}_{k}, k}=\omega_{\hat{x}_{k}}^{-1}\left(\boldsymbol{\Lambda}_{k}\right)$. Pour tout $p \in B$, l'espace $H_{p, \mathfrak{n}^{-}}$(voir notation 1) est dit espace horizontal en $p$ (cette distribution n'est pas invariante pour l'action de $P$ sur $B$, contrairement au cas des connexions au sens de Ehresmann).

Soient $x \in M, \hat{x} \in B$ au-dessus de $x$, et $\gamma \in C^{1}(M, I, x)$ telle que $\mathcal{D}_{x}^{\hat{x}}(\gamma) \subset \Omega_{o}$. Il existe alors un unique remonté horizontal de $\gamma$ dans $C^{1}(B, I, \hat{x})$ (i.e. de vecteur tangent horizontal pour tout $t \in I)$. En effet, soit $\hat{\gamma}$ un remonté quelconque de $\gamma$ dans $C^{1}(B, I, \hat{x})$, et $\hat{\alpha}=\hat{\mathcal{D}}_{\hat{x}}(\hat{\gamma})$. Comme $N^{-}$est transverse aux fibres de $G \rightarrow \mathbf{X}$ au-dessus de $\Omega_{o}$, il existe $a \in C^{1}(P, I, e)$ tel que $R_{a} \hat{\alpha}$ soit une courbe de $C^{1}\left(N^{-}, I, e\right)$. Il résulte alors du lemme 5, point (ii), que $R_{a} \hat{\gamma}$ est également horizontale.

Revenons à présent à la preuve du lemme 7. Supposons dans un premier temps que $\boldsymbol{\Lambda}_{k}$ est juste une suite de points $\xi_{k}$ dans $\mathbf{Q} \cap \mathbf{W}_{\hat{x}_{k}}$. D'après les remarques précédentes, il existe une

$4^{\text {e }}$ SÉRIE - TOME $40-2007-\mathrm{N}^{\circ} 5$ 
courbe horizontale $\hat{\gamma}_{k} \in C^{1}\left(B, I, \hat{x}_{k}\right)$ au-dessus de $\xi_{\hat{x}_{k}, k}^{*}$. La courbe $\hat{\alpha}_{k}=\hat{\mathcal{D}}_{\hat{x}_{k}}\left(\hat{\gamma}_{k}\right)$ est alors une courbe de $C^{1}\left(N^{-}, I, e\right)$ qui se projette sur $\xi_{k}^{*}$. On remarque que, si $\left(a_{1, k}(t), \ldots, a_{n, k}(t)\right)$ sont les coordonnées de $\hat{\gamma}_{k}^{\prime}(t)$ relativement au parallélisme $\mathcal{R}$, alors les coordonnées de $\hat{\alpha}_{k}^{\prime}(t)$ relativement au parallélisme défini par $E_{1}, \ldots, E_{n}$ sur $T G$ sont également $\left(a_{1, k}(t), \ldots, a_{n, k}(t)\right)$. On obtient ainsi que $L_{\rho}\left(\hat{\gamma}_{k}\right)=L_{\rho_{G}}\left(\hat{\alpha}_{k}\right)=L^{-}\left(\xi_{k}^{*}\right)$. Grâce aux hypothèses du lemme, et à la propriété $\left(P_{2}\right)$ du lemme 2 , nous obtenons : $\lim _{k \rightarrow+\infty} L_{\rho}\left(\hat{\gamma}_{k}\right)=0$. Ainsi, pour $k$ suffisamment grand, $\left[\hat{\gamma}_{k}\right]$ est inclus dans toute $\rho$-boule, de rayon arbitrairement petit, et centrée en $\hat{x}_{\infty}$. En projetant, on obtient que, pour tout voisinage de $x_{\infty},\left[\xi_{\hat{x}_{k}, k}\right]$ est inclus dans ce voisinage dès que $k$ est suffisamment grand.

Maintenant dans le cas général, $\left[\Lambda_{\hat{x}_{k}, k}\right]$ tend vers $\left[x_{\infty}\right]$ si et seulement si pour toute suite $\sigma_{k}$ de $\Lambda_{\hat{x}_{k}, k},\left[\sigma_{k}\right]$ tend vers $\left[x_{\infty}\right]$. On est donc ramené à la situation précédente.

Nous finissons cette section avec le lemme technique suivant :

Lemme 8. - Soient $x \in M$ et $\hat{x}$ un point de $B$ au-dessus de $x$. Il existe un ouvert $U \subset H_{\hat{x}, \mathfrak{z}^{-}}$ contenant $0_{\hat{x}}$ tel que $U \backslash\left\{0_{\hat{x}}\right\} \subset Q_{\hat{x}}^{\text {reg }}$.

Preuve. - On commence par remarquer que, pour tout $\xi \in \mathfrak{z}^{-} \backslash\left\{0_{\mathfrak{g}}\right\}, \mathfrak{n}^{-} \subset T_{\xi} \dot{\mathbf{Q}}$. C'est complètement évident si $\mathfrak{z}^{-}=\mathfrak{n}^{-}$(i.e $\mathfrak{g}=\mathfrak{s o}(1, n), \mathfrak{s u}(1,1)$ ou $\left.\mathfrak{s p}(1,1)\right)$. Dans les autres cas, on fixe $\zeta_{1}, \ldots, \zeta_{s}$ une base de $\mathfrak{n}_{1}^{+}$. Le flot $\exp _{G}(t \xi)$ ne commute à aucun des $\operatorname{Exp}_{G}\left(t \zeta_{i}\right), 1 \leqslant i \leqslant s$. En effet, le premier agit sur $\mathbf{X}$ avec un seul point fixe qui est $\nu$. Les seconds agissent sur $\mathbf{X}$ avec un seul point fixe qui est $o$. Par conséquent $\left[\xi, \zeta_{1}\right], \ldots,\left[\xi, \zeta_{s}\right]$ constitue une famille libre, qui est en fait une base de $\mathfrak{n}_{1}^{-}$(en effet, on est dans le cas où $\mathfrak{z}^{-}=\mathfrak{g}_{-2 \alpha}$ et $\mathfrak{n}_{1}^{+}=\mathfrak{g}_{+\alpha}$, voir section 2.1, donc $\left.\left[\mathfrak{z}^{-}, \mathfrak{n}_{1}^{+}\right] \subset \mathfrak{n}_{1}^{-}\right)$. D' autre part, comme pour tout $1 \leqslant i \leqslant s,\left[\xi, \zeta_{i}\right]=\left.\frac{d}{d t}\right|_{t=0} A d\left(\operatorname{Exp}_{G}\left(t \zeta_{i}\right)\right) . \xi$, on a $\left[\xi, \zeta_{i}\right] \in T_{\xi} \dot{\mathbf{Q}}$. Finalement $\mathfrak{n}_{1}^{-} \subset T_{\xi} \dot{\mathbf{Q}}$, et comme par définition de $\mathbf{Q}, \mathfrak{z}^{-} \subset T_{\xi} \dot{\mathbf{Q}}$, on obtient bien $\mathfrak{n}^{-} \subset T_{\xi} \dot{\mathbf{Q}}$.

Choisissons un ouvert $V_{\hat{x}}$ contenant $0_{\hat{x}}$ tel que $\operatorname{Exp}_{\hat{x}}$ réalise un difféomorphisme de $V_{\hat{x}}$ sur son image. L'image réciproque dans $V_{\hat{x}}$ des fibres de $B \rightarrow M$ donne un feuilletage $\mathcal{F}$ de $V_{\hat{x}}$. La feuille $\mathcal{F}_{0_{\hat{x}}}$ passant par $0_{\hat{x}}$ n'est autre que $H_{\hat{x}, \mathfrak{p}} \cap V_{\hat{x}}$, et en particulier est transverse à $H_{\hat{x}, \mathfrak{n}^{-}}$. Donc il existe un voisinage $U$ de $0_{\hat{x}}$ dans $H_{\hat{x}, \mathfrak{z}^{-}}$tel que, pour tout $u \in U, \mathcal{F}_{u}$ est transverse à $H_{\hat{x}, \mathfrak{n}^{-}}$. Maintenant, par ce qui vient d'être dit plus haut, pour tout $u \in U \backslash\left\{0_{\hat{x}}\right\}, H_{\hat{x}, \mathfrak{n}^{-}} \subset T_{u} \dot{Q}_{\hat{x}}$. Ainsi, la différentielle de $\operatorname{Exp}_{x}$ en restriction à $T_{u} \dot{Q}_{\hat{x}}$ est bien une surjection sur $T_{\operatorname{Exp}_{x}(u)} M$.

\section{Automorphismes d'une géométrie de Cartan}

\subsection{Remarques préliminaires}

Dans la première partie de cette section, nous considérons une géométrie de Cartan générale, sans faire d'hypothèse particulière sur les groupes $G$ et $P$. Le groupe $A u t(B, \omega)$ a été défini dans l'introduction de l'article : il est constitué des difféomorphismes $h$ de $B$ tels que $h^{*} \omega=\omega$. Tout élément de $\operatorname{Aut}(B, \omega)$ laisse le parallélisme $\mathcal{R}$ invariant, et par conséquent, $A u t(B, \omega)$ est un sous-groupe fermé de $\operatorname{Iso}(B, \rho)$. Ce dernier est lui-même fermé dans $H o m e o(B)$, d'après les propriétés classiques du groupe d'isométries d'une variété riemannienne (voir par exemple $[16,25])$.

\subsection{Suites d'holonomie}

DÉFInItion 11 (Donnée d'holonomie). - Soit $\left(f_{k}, x_{k}, x_{\infty}, y_{\infty}\right)$ une donnée stable de $\operatorname{Aut}(M, \omega)$. On dit que $\left(b_{k}, \hat{x}_{k}, \hat{x}_{\infty}, \hat{y}_{\infty}\right)$ est une donnée d'holonomie associée, s'il existe des 
remontés $\hat{f}_{k}$ des $f_{k}$ dans $A u t(B, \omega)$, une suite $\hat{x}_{k}$ de $B$ au-dessus de $x_{k}$, qui tend vers $\hat{x}_{\infty}$, et une suite $\hat{y}_{\infty} \in B$ au-dessus de $y_{\infty}$, de sorte que $\hat{y}_{k}=R_{b_{k}^{-1}} \circ \hat{f}_{k}\left(\hat{x}_{k}\right)$ tende vers $\hat{y}_{\infty}$.

Pour toute donnée stable $\left(f_{k}, x_{k}, x_{\infty}, y_{\infty}\right)$ de $\operatorname{Aut}(M, \omega)$, il est toujours possible d'associer une donnée d'holonomie $\left(b_{k}, \hat{x}_{k}, \hat{x}_{\infty}, \hat{y}_{\infty}\right)$. Pour le voir, on se fixe deux ouverts suffisamment petits autour de $x_{\infty}$ et $y_{\infty}$ respectivement, de sorte qu'il existe des sections continues $s_{U}: U \rightarrow B$ et $s_{V}: V \rightarrow B$. Soient $\hat{f}_{k}$ des remontés des $f_{k}$ dans $A u t(B, \omega)$. On pose alors $s_{U}\left(x_{k}\right)=\hat{x}_{k}$, $k \in \mathbf{N} \cup\{\infty\}$ (resp. $s_{V}\left(f_{k}\left(x_{k}\right)\right)=\hat{y}_{k}, k \in \mathbf{N} \cup\{\infty\}$ ). D' autre part, comme $\hat{f}_{k}\left(\hat{x}_{k}\right)$ est une suite au-dessus de $f_{k}\left(x_{k}\right)$, il existe une unique suite $\left(b_{k}\right)$ d'éléments de $P$, telle que pour tout $k \in \mathbf{N}$, on ait $R_{b_{k}^{-1}} \circ \hat{f}\left(\hat{x}_{k}\right)=\hat{y}_{k}$.

Proposition 3 (Équivariance). - Soient $\left(f_{k}, x_{k}, x_{\infty}, y_{\infty}\right)$ une donnée stable de Aut $(M, \omega)$, et $\left(b_{k}, \hat{x}_{k}, \hat{x}_{\infty}, \hat{y}_{\infty}\right)$ une holonomie associée. Pour toute suite $\xi_{k} \in \mathbf{W}_{\hat{x}_{k}}$, on note $\zeta_{k}=$ $A d\left(b_{k}\right) . \xi_{k}$. On a alors la relation d'équivariance:

$$
f_{k}\left(\xi_{\hat{x}_{k}, k}^{*}\right)=\zeta_{\hat{y}_{k}, k}^{*}
$$

Preuve. - Soient $\hat{f}_{k}$ les remontés des $f_{k}$ qui permettent de définir la suite d'holonomie $\left(b_{k}\right)$. On appelle $\hat{\phi}_{k}=R_{b_{k}^{-1}} \circ \hat{f}_{k}$. Par définition, $\phi_{k}\left(\hat{x}_{k}\right)=\hat{y}_{k}$ et $\lim _{k \rightarrow+\infty} \hat{y}_{k}=\hat{y}_{\infty}$. On observe également que $\left(\hat{\phi}_{k}\right)_{*} \xi^{\dagger}=\left(A d\left(b_{k}\right) \xi\right)^{\dagger}$. Cela nous permet d'obtenir tout d'abord que si $\xi_{k} \in \mathbf{W}_{\hat{x}_{k}}$, alors $\zeta_{k} \in \mathbf{W}_{\hat{y}_{k}}$, puis que $\hat{\phi}_{k}\left(\hat{\xi}_{\hat{x}_{k}, k}^{*}\right)=\hat{\zeta}_{\hat{y}_{k}, k}^{*}$. En projetant sur $M$, on obtient la relation (4).

On considère maintenant une géométrie de Cartan $(M, B, \omega)$ modelée sur un espace $\mathbf{X}=$ $\partial \mathbf{H}_{\mathbb{K}}^{d}$.

THÉORÈME 3. - (i) Le groupe Aut $(M, \omega)$ est fermé dans Homeo( $M)$.

Soit $\left(f_{k}, x_{k}, x_{\infty}, y_{\infty}\right)$ une donnée stable de $\operatorname{Aut}(M, \omega)$, et $\left(b_{k}, \hat{x}_{k}, \hat{x}_{\infty}, \hat{y}_{\infty}\right)$ une holonomie associée. Alors :

(ii) $\left(f_{k}\right)$ est bornée dans Aut $(M, \omega)$ si et seulement si $\left(b_{k}\right)$ est bornée dans $G$.

(iii) Si la suite $\left(f_{k}\right)$ agit équicontinûment en $x_{\infty}$, alors $\left(b_{k}\right)$ agit équicontinûment en 0 .

Preuve. - On commence par démontrer le point (iii).

Supposons qu'au contraire, $\left(b_{k}\right)$ n'agisse pas équicontinûment en $o$. Il est alors possible de choisir une suite $\left(z_{k}\right)$ de $\mathbf{X}$, qui tend vers $o$, et telle que $w_{k}=b_{k} . z_{k}$ tende vers $w_{\infty} \neq o$. Par la propriété $\left(P_{1}\right)$ du lemme 2, il existe, pour tout $k$, un segment géodésique $\alpha_{k}=\left[z_{k}, u_{k}\right)$ reliant $o$ à $z_{k}$ (on identifie toujours $[\mathbf{Q}]$ avec $\mathfrak{n}^{+} \times S_{\mathfrak{z}^{+}}$). On choisit les $\alpha_{k}$ de sorte que $\lim _{k \rightarrow+\infty} \alpha_{k}=[o]$. Quitte à extraire une sous-suite, on peut supposer que la suite $\beta_{k}=b_{k} . \alpha_{k}$ tend vers $\beta_{\infty}=\left[w_{\infty}, u_{\infty}\right)$. On pose $\xi_{k}=s\left(\alpha_{k}\right)$ et $\zeta_{k}=s\left(\beta_{k}\right)$. On pose aussi $\zeta_{\infty}=s\left(\beta_{\infty}\right)$, et $\lim _{k \rightarrow+\infty} \zeta_{k}=\zeta_{\infty}$ par continuité de $s$. D'autre part, on peut trouver un réel $\left.\left.\lambda \in\right] 0,1\right]$ tel que $\lambda \zeta_{k} \in \mathbf{W}_{\hat{y}_{k}}$ pour tout $k \in \mathbf{N} \cup\{\infty\}$. Ainsi, quitte à remplacer $\zeta_{k}$ par $\lambda \zeta_{k}$, et $\xi_{k}$ par $A d\left(b_{k}^{-1}\right)\left(\lambda \zeta_{k}\right)$, on peut supposer que $\xi_{k} \in \mathbf{W}_{\hat{x}_{k}}$ pour tout $k \in \mathbf{N}$, et $\zeta_{k} \in \mathbf{W}_{\hat{y}_{k}}$ pour tout $k \in \mathbf{N} \cup\{\infty\}$.

De la proposition 3, on déduit pour tout $k \in \mathbf{N}$, et $t \in[0,1]: f_{k}\left(\xi_{\hat{x}_{k}, k}^{*}(t)\right)=\zeta_{\hat{y}_{k}, k}^{*}(t)$. Comme $\zeta_{\hat{y}_{\infty}, \infty} \neq 0$, il existe $t_{0} \in[0,1]$ tel que $\zeta_{\hat{y}_{\infty}, \infty}^{*}\left(t_{0}\right) \neq y_{\infty}$. Ainsi, $\lim _{k \rightarrow+\infty} f_{k}\left(\xi_{\hat{x}_{k}, k}^{*}\left(t_{0}\right)\right) \neq y_{\infty}$. Pourtant, $\xi_{\hat{x}_{k}, k}^{*}\left(t_{0}\right)$ tend vers $x_{\infty}$. En effet, $\lim _{k \rightarrow+\infty}\left[\xi_{k}\right]=[o]$ et le lemme 7 implique alors $\lim _{k \rightarrow+\infty}\left[\xi_{\hat{x}_{k}, k}\right]=\left[x_{\infty}\right]$. L'action de $\left(f_{k}\right)$ n'est donc pas équicontinue en $x_{\infty}$.

On peut à présent démontrer le point (i). On suppose que $\left(f_{k}\right)$ est une suite de $\operatorname{Aut}(M, \omega)$ qui converge vers $f_{\infty} \in \operatorname{Homeo}(M)$. Alors pour tout point $x \in M$, si $\left(x_{k}\right)$ désigne la suite constante égale à $x$ et $y_{\infty}=f_{\infty}(x)$, le quadruplet $\left(f_{k}, x_{k}, x, y_{\infty}\right)$ est une donnée stable. On note $\left(b_{k}, \hat{x}_{k}, \hat{x}, \hat{y}_{\infty}\right)$ une holonomie associée. On prouve alors le :

$4^{e}$ SÉRIE - TOME $40-2007-\mathrm{N}^{\circ} 5$ 
Lemme 9. - La suite $\left(b_{k}\right)$ est bornée dans $G$.

Preuve. - Dans le cas contraire, il existe une sous-suite de $\left(b_{k}\right)$ qui admet une dynamique Nord-Sud de pôles $o^{+}$et $o^{-}$. Comme $o$ est fixé par $\left(b_{k}\right)$, on a nécessairement $o=o^{+}$ou $o=o^{-}$. Dans le second cas, l'action de $\left(b_{k}\right)$ n'est pas équicontinue en $o$ (voir corollaire 1), et il résulte du point (iii) que celle de $\left(f_{k}\right)$ ne devrait pas l'être en $x$. Or ceci est absurde puisque $\left(f_{k}\right)$ admet une limite dans $\operatorname{Homeo}_{(M)}$. On est donc dans le cas $o=o^{+}$. On se donne alors $\alpha=[\xi]$ un segment de $[\dot{\mathbf{Q}}]$, tel que $\xi \in \mathbf{W}_{\hat{x}}$, et $\alpha \subset \Omega_{o}$. Notons qu'alors $\zeta_{k}=A d\left(b_{k}\right) . \xi \in \mathbf{W}_{\hat{y}_{k}}$ pour tout $k \in \mathbf{N}$. Étant donné que $o=o^{+}$, on a $\lim _{k \rightarrow+\infty} b_{k} \cdot \alpha=[o]$. Ainsi, par le lemme 7, on obtient $\lim _{k \rightarrow+\infty}\left[\zeta_{\hat{y}_{k}, k}\right]=\left[y_{\infty}\right]$. Mais la proposition 3 donne également $f_{k}\left(\left[\xi_{\hat{x}_{k}}\right]\right)=\left[\zeta_{\hat{y}_{k}, k}\right]$ pour tout $k \in \mathbf{N}$, ce qui implique en passant à la limite $f_{\infty}\left(\left[\xi_{\hat{x}}\right]\right)=\left[y_{\infty}\right]$. Comme $\left[\xi_{\hat{x}}\right] \neq[x]$, on obtient une contradiction avec le fait que $f_{\infty} \in \operatorname{Homeo~}(M)$.

On réextrait à nouveau une suite de $\left(f_{k}\right)$, de sorte que $\left(b_{k}\right)$ tende vers $b_{\infty} \in P$. Cela signifie que $\hat{f}_{k}\left(\hat{x}_{k}\right)$ tend vers $R_{b_{\infty}} . \hat{y}_{\infty}$. Or $\left(\hat{f}_{k}\right)$ est une suite d'isométries pour la métrique riemannienne $\rho$. Par propreté de l'action du groupe d'isométries d'une variété riemannienne, $\hat{f}_{k}$ est relativement compacte dans $\operatorname{Iso}(B, \rho)$. Comme $A u t(B, \omega)$ est fermé dans $\operatorname{Iso}(B, \rho)$, on peut supposer, en extrayant encore, qu'il existe $\hat{f}_{\infty} \in \operatorname{Aut}(B, \omega)$ tel que $\hat{f}_{k} \rightarrow \hat{f}_{\infty}$. Finalement, $\hat{f}_{\infty}$ est un relevé de $f_{\infty}$ dans $A u t(B, \omega)$, et par conséquent $f_{\infty} \in \operatorname{Aut}(M, \omega)$.

La preuve du point (ii) se fait de manière analogue à celle du point (i).

Remarque 3. - Il est probable que l'usage des géodésiques et des suites d'holonomies puisse se généraliser, pour montrer que le groupe des automorphismes de toute géométrie de Cartan parabolique sur $M$ est fermé dans Homeo $(M)$.

\section{Preuve du théorème 1}

On commence par montrer une version facile du théorème 1 pour les variétés kleiniennes, c'est-à-dire des variétés qui sont quotient d'un ouvert $\Omega \subset \mathbf{X}$ par un sous-groupe discret $\Gamma \subset G$. Ces variétés sont naturellement munies d'une géométrie de Cartan, où $B=\Gamma \backslash \pi_{X}^{-1}(\Omega)$, et $\omega=\bar{\omega}_{G}$, la connexion de Cartan induite par la forme $\omega_{G}$ sur ce quotient.

LEMME 10. - Soient $\Omega$ un ouvert connexe de $\mathbf{X}$, et $M=\Gamma \backslash \Omega$, où $\Gamma \subset G$ est un sous-groupe discret agissant proprement discontinûment sur $\Omega$.

Si Aut $\left(M, \bar{\omega}_{G}\right)$ n'agit pas proprement sur $M$, alors $\Gamma=\{e\}$ et :

- ou bien $\Omega=\mathbf{X} \backslash\{\kappa\}$, pour un certain $\kappa \in \mathbf{X}$.

- ou bien $\Omega=\mathbf{X}$.

Preuve. - Le groupe $\operatorname{Aut}\left(M, \bar{\omega}_{G}\right)$ est induit par les éléments de $G$ qui laissent $\Omega$ stable et normalisent $\Gamma$. Par conséquent, si $\operatorname{Aut}\left(M, \bar{\omega}_{G}\right)$ n'agit pas proprement sur $M$, on peut trouver une suite $\left(h_{k}\right)$ de $G$ qui normalise $\Gamma$, et qui n'agit pas proprement sur $\Omega$. On peut supposer que cette suite tend vers l'infini dans $G$, et quitte à considérer $\left(h_{k}^{-1}\right)$ à la place de $\left(h_{k}\right)$, on suppose également $o^{-} \in \Omega$. On appelle $\pi_{M}$ l'application de revêtement de $\Omega$ sur $M$, et on considère un petit ouvert $U \subset \Omega$ contenant $o^{-}$tel que $\pi_{M}$ soit un difféomorphisme de $U$ sur son image. Par le lemme 4, il existe une suite $\left(k_{m}\right)_{m \in \mathbf{N}}$ telle que $h_{k_{m}}(U)$ soit une suite croissante d'ouverts dont la réunion est $\mathbf{X}$ si $o^{+}=o^{-}$et $\mathbf{X} \backslash\left\{o^{+}\right\}$si $o^{+} \neq o^{-}$. On en conclut que $\Omega=\mathbf{X}$ ou $\Omega=\mathbf{X} \backslash\left\{o^{+}\right\}$. D'autre part, comme $h_{k}$ normalise $\Gamma$ pour tout $k, \pi_{M}$ doit être injective sur chaque $h_{k_{m}}(U)$. Au final, $\pi_{M}$ est injective sur un ouvert dense de $\Omega$, ce qui entraîne que $\Gamma=\{e\}$.

Nous nous plaçons à présent sous les hypothèses générales du théorème $1:(M, B, \omega)$ est une géométrie de Cartan modelée sur $\mathbf{X}=\partial \mathbf{H}_{\mathbb{K}}^{d}$, $\omega$ est une connexion régulière, et $\operatorname{Aut}(M, \omega)$ n'agit pas proprement sur $M$. 
Proposition 4. - Si $\left(f_{k}\right)$ est une suite de Aut $(M, \omega)$ qui n'agit pas proprement sur $M$, alors il existe un ouvert $U \in M$ et un point $y_{\infty} \in M$, tels que $\lim _{k \rightarrow+\infty} f_{k}(U)=y_{\infty}$.

Preuve. - Comme $\operatorname{Aut}(M, \omega)$ n'agit pas proprement sur $M$, il existe une donnée stable $\left(f_{k}, x_{k}, x_{\infty}, y_{\infty}\right)$, avec $\left(f_{k}\right)$ une suite de $\operatorname{Aut}(M, \omega)$ qui tend vers l'infini. On note $\left(b_{k}, \hat{x}_{k}, \hat{x}_{\infty}, \hat{y}_{\infty}\right)$ une holonomie associée. Par le théorème 3 , la suite $\left(b_{k}\right)$ tend vers l'infini dans $P$, et quitte à changer $\left(f_{k}, x_{k}, x_{\infty}, y_{\infty}\right)$ en la donnée stable $\left(f_{k}^{-1}, y_{k}, y_{\infty}, x_{\infty}\right)$, le lemme 2 assure qu'il existe un ouvert $\boldsymbol{\Omega}^{+} \subset[\dot{\mathbf{Q}}]$, tel que pour tout compact $K \subset \boldsymbol{\Omega}^{+}, \lim _{k \rightarrow+\infty} b_{k} . K=[o]$. Soit $\mathbf{U}^{+}=s\left(\boldsymbol{\Omega}^{+}\right)$. Pour tout $p \in B$, on note $U_{p}^{+}=\omega_{p}^{-1}\left(\mathbf{U}^{+}\right)$.

D'après le lemme 8 et le point (ii) de la proposition 2, il existe $\xi \in \mathbf{U}^{+}$tel que $\xi_{\hat{x}_{\infty}} \in \dot{Q}_{\hat{x}_{\infty}}^{\text {reg }}$. En fait, il existe $\epsilon>0$ petit tel que $B_{\hat{x}_{\infty}}(\epsilon) \subset \dot{Q}_{\hat{x}_{\infty}}^{\text {reg }}$, où $B_{\hat{x}_{\infty}}(\epsilon)=\omega_{\hat{x}_{\infty}}^{-1}\left(B_{\rho_{G}}(\xi, \epsilon) \cap \mathbf{U}^{+}\right)$. Cette propriété reste vraie si l'on remplace $x_{\infty}$ par $x_{k}$ pour $k \geqslant k_{0}$ suffisamment grand, c'est-à-dire que si l'on pose $B_{\hat{x}_{k}}(\epsilon)=\omega_{\hat{x}_{k}}^{-1}\left(B_{\rho_{G}}(\xi, \epsilon) \cap \mathbf{U}^{+}\right)$, alors pour $k \geqslant k_{0}, U_{k}=\operatorname{Exp}_{x_{k}}\left(B_{\hat{x}_{k}}(\epsilon)\right)$ est une suite d'ouverts de $M$. De plus, cette suite converge vers l'ouvert $U_{\infty}=\operatorname{Exp}_{x_{\infty}}\left(B_{\hat{x}_{\infty}}(\epsilon)\right)$. Il existe donc un ouvert $U \subset M$, et un entier $k_{1} \geqslant k_{0}$, tels que $U \subset \bigcap_{k \geqslant k_{1}} U_{k}$.

Maintenant, on suppose $\epsilon$ suffisamment petit pour que $B_{\rho_{G}}(\xi, \epsilon) \cap \mathbf{U}^{+}$soit d'adhérence compacte dans $\mathbf{U}^{+}$, et on pose $\boldsymbol{\Lambda}_{k}=A d\left(b_{k}\right) \cdot\left(B_{\rho_{G}}(\xi, \epsilon) \cap \mathbf{U}^{+}\right)$. On a alors $\lim _{k \rightarrow+\infty}\left[\boldsymbol{\Lambda}_{k}\right]=$ $\lim _{k \rightarrow+\infty} b_{k} \cdot\left[B_{\rho_{G}}(\xi, \epsilon) \cap \mathbf{U}^{+}\right]=[o]$. D'autre part $\left[\Lambda_{x_{k}, k}\right]=f_{k}\left(\left[B_{\hat{x}_{k}}(\epsilon)\right]\right)$, comme conséquence de la relation (4) de la proposition 3. Le lemme 7 assure alors que $\lim _{k \rightarrow+\infty} f_{k}\left(\left[B_{\hat{x}_{k}}(\epsilon)\right]\right)=$ $\left[y_{\infty}\right]$. Comme $\operatorname{Exp}_{x_{k}}\left(B_{\hat{x}_{k}}(\epsilon)\right) \subset\left[B_{\hat{x}_{k}}(\epsilon)\right]$, on a $U \subset\left[B_{\hat{x}_{k}}(\epsilon)\right]$ pour tout $k \geqslant k_{1}$, et la proposition en découle.

Proposition 5. - Soient $\left(f_{k}\right)$ une suite de Aut $(M, \omega)$ qui tend vers l'infini, et $\left(f_{k}, x_{k}, x_{\infty}, y_{\infty}\right)$ une donnée stable sur $M$. Si l'action de $\left(f_{k}\right)$ est équicontinue en $x_{\infty}$, alors la courbure de $\omega$ est nulle en $x_{\infty}$.

Preuve. - Soit $\left(b_{k}, \hat{x}_{k}, \hat{x}_{\infty}, \hat{y}_{\infty}\right)$ une donnée d'holonomie associée à $\left(f_{k}, x_{k}, x_{\infty}, y_{\infty}\right)$. Par le théorème 3 , la suite $\left(b_{k}\right)$ est équicontinue en $o$. On applique alors les résultats du corollaire 1 : les pôles attracteurs et répulseurs de $\left(b_{k}\right)$ satisfont $o^{+}=o$ et $o^{-} \neq o$, et l'on peut écrire $b_{k}=l_{1, k} a^{t_{k}} l_{2, k}$, où $l_{1, k}$ et $l_{2, k}$ sont deux suites relativement compactes de $P$. On supposera que, pour $i \in\{1,2\}, l_{i, k}$ converge vers $l_{i, \infty} \in P$. D'autre part, comme $o^{+}=o$, on doit avoir $\lim _{k \rightarrow+\infty} t_{k}=+\infty$.

Fixons-nous une base $\eta_{1}, \ldots, \eta_{n}$ de $\mathfrak{n}^{-}$. On suppose que $\{1, \ldots, n\}$ se décompose en $I_{-1} \cup I_{-2}$, tel que $\eta_{i} \in \mathfrak{n}_{1}^{-}$pour $i \in I_{-1}$, et $\eta_{i} \in \mathfrak{z}^{-}$pour $i \in I_{-2}$ (bien sûr $I_{-1}=\emptyset$ lorsque $\mathfrak{n}_{1}^{-}=\{0\}$ ). Pour $i=1, \ldots, n$, on pose $\xi_{i, k}=A d\left(l_{2, k}^{-1}\right) \cdot \eta_{i}$ (resp. $\left.\zeta_{i, k}=A d\left(l_{1, k}\right) \cdot \eta_{i}\right)$. Pour $i=1, \ldots, n, \lim _{k \rightarrow+\infty} \xi_{i, k}=\xi_{i, \infty}\left(\right.$ resp. $\left.\lim _{k \rightarrow+\infty} \zeta_{i, k}=\zeta_{i, \infty}\right)$, avec $\xi_{i, \infty}=A d\left(l_{2, \infty}^{-1}\right) \cdot \eta_{i}$ (resp. $\left.\zeta_{i, \infty}=A d\left(l_{1, \infty}\right) \cdot \eta_{i}\right)$. Dans la suite, on notera $\mathfrak{h}_{k}$ (resp. $\left.\mathfrak{h}_{\infty}\right)$ le sous-espace vectoriel de $\mathfrak{g}$ engendré par $\xi_{1, k}, \ldots, \xi_{n, k}$ (resp. $\left.\xi_{1, \infty}, \ldots, \xi_{n, \infty}\right)$. Puisque $\mathfrak{h}_{k}$ n'est rien d'autre que l'image de $\mathfrak{n}^{-}$ $\operatorname{par} A d\left(l_{2, k}^{-1}\right)$, les $\mathfrak{h}_{k}$ (resp. $\left.\mathfrak{h}_{\infty}\right)$ sont des sous-algèbres de Lie de $\mathfrak{g}$.

Soit $\left(\hat{f}_{k}\right)$ le remonté de $\left(f_{k}\right)$ qui définit l'holonomie $\left(b_{k}\right)$, i.e telle que $R_{b_{k}^{-1}} \circ \hat{f}_{k}\left(\hat{x}_{k}\right)=\hat{y}_{k}$. Posons $\phi_{k}=R_{b_{k}^{-1}} \circ \hat{f}_{k}$. Alors, $\left(\phi_{k}\right)^{*} \Omega=A d\left(b_{k}\right) . \Omega$. On distingue à présent deux cas.

a) Si les racines de $\mathfrak{g}$ sont $\Delta=\{-\alpha, \alpha\}$. Alors, pour tout $k \in \mathbf{N}$, et $1 \leqslant i \leqslant n$, on a $A d\left(b_{k}\right) \cdot \xi_{i, k}=e^{-t_{k}} \zeta_{i, k}$. On en déduit la relation $D_{\hat{x}_{k}} \phi_{k}\left(\xi_{\hat{x}_{k}, i, k}\right)=e^{-t_{k}} \zeta_{\hat{y}_{k}, i, k}$, qui implique pour tout $1 \leqslant i \leqslant j \leqslant n$ :

$$
\operatorname{Ad}\left(b_{k}\right) . \Omega_{\hat{x}_{k}}\left(\xi_{\hat{x}_{k}, i, k}, \xi_{\hat{x}_{k}, j, k}\right)=e^{-2 t_{k}} \Omega_{\hat{y}_{k}}\left(\zeta_{\hat{y}_{k}, i, k}, \zeta_{\hat{y}_{k}, j, k}\right) .
$$

$4^{\mathrm{e}}$ SÉRIE - TOME $40-2007-\mathrm{N}^{\circ} 5$ 
En regardant la décomposition de $\mathfrak{g}$ en espaces de racines, on voit qu'il existe $C>0$ tel que pour tout $u \in \mathfrak{g},\left\|A d\left(b_{k}\right) . u\right\| \geqslant C e^{-t_{k}} .\|u\|$. On en déduit que

$$
\Omega_{\hat{x}_{\infty}}\left(\xi_{\hat{x}_{\infty}, i, \infty}, \xi_{\hat{x}_{\infty}, j, \infty}\right)=\lim _{k \rightarrow+\infty} \Omega_{\hat{x}_{k}}\left(\xi_{\hat{x}_{k}, i, k}, \xi_{\hat{x}_{k}, j, k}\right)=0 .
$$

Le sous-espace $\mathfrak{h}_{\infty}$ est un supplémentaire de $\mathfrak{p}$ dans $\mathfrak{g}$, si bien que $\Omega_{\hat{x}_{\infty}}=0$.

b) $\mathrm{Si}$ les racines de $\mathfrak{g}$ sont $\Delta=\{-2 \alpha,-\alpha, \alpha,+2 \alpha\}$. Alors, $A d\left(b_{k}\right) \cdot \xi_{i, k}=e^{-t_{k}} \zeta_{i, k}$ pour tout $i \in I_{-1}$, et $A d\left(b_{k}\right) \cdot \xi_{i, k}=e^{-2 t_{k}} \zeta_{i, k}$ pour tout $i \in I_{-2}$. Par les mêmes arguments que précédemment, on obtient :

- $\left\|A d\left(b_{k}\right) \cdot \Omega_{\hat{x}_{k}}\left(\xi_{\hat{x}_{k}, i, k}, \xi_{\hat{x}_{k}, j, k}\right)\right\| \leqslant e^{-2 t_{k}} \Omega_{\hat{y}_{k}}\left(\zeta_{\hat{y}_{k}, i, k}, \zeta_{\hat{y}_{k}, j, k}\right)$ pour tout $1 \leqslant i<j \leqslant n$. - $\left\|A d\left(b_{k}\right) \cdot \Omega_{\hat{x}_{k}}\left(\xi_{\hat{x}_{k}, i, k}, \xi_{\hat{x}_{k}, j, k}\right)\right\| \leqslant e^{-3 t_{k}} \Omega_{\hat{y}_{k}}\left(\zeta_{\hat{y}_{k}, i, k}, \zeta_{\hat{y}_{k}, j, k}\right)$ si $i$ ou $j$ est dans $I_{-2}$.

On regarde à nouveau la décomposition de $\mathfrak{g}$ en espaces de racines, et on observe que les suites convergentes $\left(u_{k}\right)$ de $\mathfrak{g}$, pour lesquelles $\left\|A d\left(b_{k}\right) . u_{k}\right\|$ est contracté au moins aussi vite que $e^{-2 t_{k}}$, doivent converger dans $A d\left(l_{2, \infty}^{-1}\right) \cdot \mathfrak{z}^{-}$. De même, les suites convergentes $\left(u_{k}\right)$ telles que $\left\|A d\left(b_{k}\right) \cdot u_{k}\right\|$ soit contracté au moins aussi vite que $e^{-3 t_{k}}$ doivent converger vers $0_{\mathfrak{g}}$. On en déduit que $\Omega_{\hat{x}_{\infty}}\left(\xi_{\hat{x}_{\infty}, i, \infty}, \xi_{\hat{x}_{\infty}, j, \infty}\right)=0$ dès que $i$ ou $j$ est dans $I_{-2}$. Si $i$ et $j$ sont tous les deux dans $I_{-1}$, on obtient que $\Omega_{\hat{x}_{\infty}}\left(\xi_{\hat{x}_{\infty}, i, \infty}, \xi_{\hat{x}_{\infty}, j, \infty}\right)$ est dans $A d\left(l_{2, \infty}^{-1}\right) \cdot \mathfrak{z}^{-}$. Mais comme la connexion $\omega$ est supposée régulière, ceci n'est possible que si $\Omega_{\hat{x}_{\infty}}\left(\xi_{\hat{x}_{\infty}, i, \infty}, \xi_{\hat{x}_{\infty}, j, \infty}\right)=0$. On obtient finalement que $\Omega_{\hat{x}_{\infty}}=0$ sur $\mathfrak{h}_{\infty}$, et finalement $\Omega_{\hat{x}_{\infty}}=0$.

On se fixe à présent un point $x_{\infty}$ dans l'ouvert $U$ donné par la proposition 4. Alors, si $\left(x_{k}\right)$ est la suite constante égale à $x_{\infty}$, et si $y_{k}=f_{k}\left(x_{\infty}\right)$, alors $\left(f_{k}, x_{k}, x_{\infty}, y_{\infty}\right)$ est une donnée stable. On note cette donnée stable particulière $\left(f_{k}, x_{\infty}, x_{\infty}, y_{\infty}\right)$.

Proposition 6. - Le point $x_{\infty}$ possède un voisinage ouvert $\Lambda$, qui est géométriquement isomorphe à un quotient $\Gamma \backslash \Omega_{\nu}$, avec $\Gamma \subset P$ un sous-groupe discret (éventuellement trivial).

Preuve. - On considère une donnée d'holonomie $\left(b_{k}, \hat{x}_{\infty}, \hat{x}_{\infty}, \hat{y}_{\infty}\right)$ associée à $\left(f_{k}, x_{\infty}\right.$, $\left.x_{\infty}, y_{\infty}\right)$. On pose $\phi_{k}=R_{b_{k}^{-1}} \circ \hat{f}_{k}$, et l'on reprend les notations de la proposition 5. L'action de $\left(f_{k}\right)$ étant équicontinue en $x_{\infty}, b_{k}$ s'écrit $l_{1, k} a^{t_{k}} l_{2, k}$ avec $\lim _{k \rightarrow+\infty} t_{k}=+\infty$.

Pour tout $r \in \mathbb{R}_{+}^{*}$, et $k \in \mathbf{N} \cup\{\infty\}$, on note $B_{r, k}$ l'intersection de $\mathfrak{h}_{k}$ avec la boule de centre $0_{\mathfrak{g}}$ et de rayon $r$ (pour la norme $\|\cdot\|$ ). Pour tout $p \in B$, on pose $H_{p, \mathfrak{h}_{k}}=\omega_{p}^{-1}\left(\mathfrak{h}_{k}\right)$, et $B_{p, r, k}=\omega_{p}^{-1}\left(B_{r, k}\right)$. Puisque $\hat{x}_{k}$ et $\hat{y}_{k}$ sont toutes deux des suites convergentes, il existe $r \in \mathbb{R}_{+}^{*}$ tel que $B_{r, k} \subset \mathbf{W}_{\hat{x}_{k}} \cap \mathbf{W}_{\hat{y}_{k}}$ pour tout $k \in \mathbf{N} \cup\{\infty\}$.

LEMME 11. - Il existe une suite d'entiers $\left(k_{m}\right)_{m \in \mathbf{N}}$ vérifiant :

- pour tout $m \in \mathbf{N}, B_{\hat{x}_{\infty}, m, k_{m}} \subset W_{\hat{x}_{\infty}}^{B}$, et Exp $x_{x_{\infty}}$ est un difféomorphisme de $B_{\hat{x}_{\infty}, m, k_{m}}$ sur son image, que l'on appelle $U_{m}$,

- $V_{m}=\left[B_{m, k_{m}}\right]$ est une suite d'ouverts de $\mathbf{X} \backslash\left\{o^{-}\right\}$, strictement croissante pour l'inclusion, dont l'union est $\mathbf{X} \backslash\left\{o^{-}\right\}$

Preuve. - De la relation $\phi_{k} \circ \operatorname{Exp}_{\hat{x}_{\infty}}=\operatorname{Exp}_{\hat{y}_{k}} \circ D_{\hat{x}_{\infty}} \phi_{k}$, on déduit que $D_{\hat{x}_{\infty}} \phi_{k}$ envoie $W_{\hat{x}_{\infty}}^{B}$ sur $W_{\hat{y}_{k}}^{B}$. On fixe $m$. Puisque $A d\left(b_{k}\right)\left(B_{m, k}\right)$ tend vers $0_{\mathfrak{g}}$ lorsque $k \rightarrow+\infty, D_{\hat{x}_{\infty}} \phi_{k}\left(B_{\hat{x}_{\infty}, m, k}\right)$ tend vers $0_{\hat{y}_{\infty}}$ lorsque $k \rightarrow+\infty$. Ainsi, pour $k$ suffisamment grand, $\operatorname{Exp}_{\hat{y}_{k}}$ réalise un difféomorphisme de $D_{\hat{x}_{\infty}} \phi_{k}\left(B_{\hat{x}_{\infty}, m, k}\right)$ sur son image. Par ailleurs, comme $\operatorname{Ad}\left(l_{1, \infty}\right)\left(\mathfrak{n}^{-}\right)$est transverse à $\mathfrak{p}$, l'image de $D_{\hat{x}_{\infty}} \phi_{k}\left(B_{\hat{x}_{\infty}, m, k}\right)$ par $\operatorname{Exp}_{\hat{y}_{k}}$ est transverse aux fibres de $B$, pour $k$ plus grand qu'un certain entier $k_{1, m}$. On en déduit que $\operatorname{Exp}_{\hat{x}_{\infty}}$ est un difféomorphisme de $B_{\hat{x}_{\infty}, m, k}$ sur son image. D'autre part, lorsque $k \rightarrow+\infty,\left[B_{m, k}\right]$ tend vers $\left[B_{m, \infty}\right]$, qui est d'adhérence compacte dans $\left[\mathfrak{h}_{\infty}\right]=\mathbf{X} \backslash\left\{o^{-}\right\}$. Ainsi, en prenant $k \geqslant k_{2, m}$, on peut supposer que $\left[B_{m, k}\right] \subset \mathbf{X} \backslash\left\{o^{-}\right\}$. Finalement, puisque $\left[B_{m, \infty}\right]$ est une suite strictement croissante pour 
l'inclusion, on peut trouver une suite $\left(k_{m}\right)$, avec $k_{m} \geqslant \max \left(k_{1, m}, k_{2, m}\right)$ pour tout $m$, telle que $\left[B_{m, k_{m}}\right]$ soit également strictement croissante pour l'inclusion.

Les propriétés de la dynamique "Nord-Sud" pour la suite $\left(b_{k}\right)$ impliquent que $\lim _{k \rightarrow+\infty} b_{k}$. $\left[B_{m, k_{m}}\right]=[o]$. Le lemme 7 affirme alors que $: \lim _{k \rightarrow+\infty} f_{k}\left(\left[B_{\hat{x}_{\infty}, m, k_{m}}\right]\right)=\left[y_{\infty}\right]$. On en conclut que l'action de $\left(f_{k}\right)$ est équicontinue en chaque point $x \in U_{m}$, et par conséquent, par la proposition 5, la courbure s'annule sur $U_{m}$.

On est à présent dans la situation suivante. L'ouvert $U_{\infty}=\bigcup_{m=1}^{\infty} U_{m}$ contient $x_{\infty}$ et est plat. On considère alors $\tilde{U}_{\infty}$ son revêtement universel, $\tilde{x}_{\infty} \in \tilde{U}_{\infty}$ au-dessus de $x_{\infty}$, et $\delta: \tilde{U}_{\infty} \rightarrow \mathbf{X}$ une application développante qui envoie $\tilde{x}_{\infty}$ sur $o$ (voir la section 3.3.1). Pour chaque $m \in \mathbf{N}, U_{m}$ se remonte en un ouvert $\tilde{U}_{m} \subset \tilde{U}_{\infty}$ de la manière suivante : pour tout $\zeta \in B_{\hat{x}_{\infty}, m, k_{m}}$, la courbe $\zeta^{*}$ se remonte de manière unique en une courbe $\tilde{\zeta}^{*} \in C^{1}\left(\tilde{U}_{\infty},[0,1], \tilde{x}_{\infty}\right)$. L'ouvert $\tilde{U}_{m}$ est la réunion de ces courbes remontées. Comme pour tout $\zeta \neq \xi$ dans $B_{\hat{x}_{\infty}, m, k_{m}}, \zeta^{*}(1) \neq \xi^{*}(1)$ (en effet, l'application $\operatorname{Exp}_{x_{\infty}}$ est un difféomorphisme de $B_{\hat{x}_{\infty}, m, k_{m}}$ sur son image), la projection de $\tilde{U}_{m}$ sur $U_{m}$ est un difféomorphisme.

On utilise à présent les résultats de la section 3.3.1. Soit $\hat{x}_{\infty}^{\prime}=R_{b_{0}} \cdot \hat{x}_{\infty}$ un point tel que $\tilde{\delta}\left(\hat{x}_{\infty}^{\prime}\right)=e$. Alors $\delta\left(\tilde{\zeta}^{*}\right)=\mathcal{D}_{x_{\infty}}^{\hat{x}_{\infty}^{\prime}}\left(\zeta^{*}\right)=b_{0} \cdot \mathcal{D}_{x_{\infty}}^{\hat{x}_{\infty}}\left(\zeta^{*}\right)$, et ainsi $\delta\left(\tilde{U}_{m}\right)=b_{0} \cdot V_{m}$.

LEMME 12. - L'application $\delta$ est injective sur $\tilde{U}_{\infty}$, et réalise un isomorphisme géométrique de $\tilde{U}_{\infty}$ dans $\mathbf{X} \backslash\left\{b_{0} . O^{-}\right\}$.

Preuve 1. - On commence par montrer que, pour tout $m \in \mathbf{N}, \delta$ est injective sur chaque $\tilde{U}_{m}$. Pour cela, prenons $\tilde{x}$ et $\tilde{y}$ distincts dans $\tilde{U}_{m}$. Leurs projetés $x$ et $y$ sont alors deux points distincts de $U_{m}$. Ils s'écrivent $x=\operatorname{Exp}_{x_{\infty}}\left(\zeta_{\hat{x}_{\infty}, m, k_{m}}\right)$ et $y=\operatorname{Exp}_{x_{\infty}}\left(\xi_{\hat{x}_{\infty}, m, k_{m}}\right)$ avec $\zeta \neq \xi$ dans $B_{m, k_{m}}$. Ainsi, $\delta(x)=\zeta^{*}(1)$ et $\delta(y)=\xi^{*}(1)$. Or $\pi_{X} \circ \operatorname{Exp}_{G}$ est un difféomorphisme de $B_{m, k_{m}}$ sur son image. Par conséquent $\delta(x) \neq \delta(y)$.

Il ne reste plus qu'à remarquer que $\tilde{U}_{m}$ est une suite croissante d'ouverts pour conclure.

Il résulte du lemme précédent, que $\tilde{U}_{\infty}$ est géométriquement isomorphe à l'ouvert $\mathbf{X} \backslash$ $\left\{b_{0} . O^{-}\right\}$, lui-même géométriquement isomorphe à $\Omega_{\nu}$. Par conséquent, $U_{\infty}$ est géométriquement isomorphe à un quotient $\Gamma \backslash \Omega_{\nu}$, où $\Gamma \subset P$ est un sous-groupe discret qui agit proprement discontinûment sans point fixe sur $\Omega_{\nu}$.

On conclut alors la preuve du théorème 1 grâce au lemme 10, et au théorème suivant, appliqué à l'inclusion $U_{\infty} \subset M$.

THÉORÈme 4. - Soit $(M, B, \omega)$ une géométrie de Cartan modelée sur $\mathbf{X}=\partial \mathbf{H}_{\mathbb{K}}^{d}$. Soit $\Gamma$ un sous-groupe discret de $P$ qui agit proprement discontinûment sans point fixe sur $\Omega_{\nu}$. On suppose qu'il existe un plongement géométrique $\sigma$ de $\Gamma \backslash \Omega_{\nu}$ dans $M$. Alors on est dans l'un des deux cas suivants :

(i) Si le groupe $\Gamma$ est trivial, $M$ est géométriquement isomorphe à l'espace modèle $\mathbf{X}$, et il existe un point $\kappa \in M$ tel que $\sigma\left(\Omega_{\nu}\right)=M \backslash\{\kappa\}$.

(ii) Si $\Gamma$ n'est pas trivial, $\sigma$ est un isomorphisme géométrique de $\Gamma \backslash \Omega_{\nu}$ sur $M$.

La preuve de ce résultat fera l'objet de la dernière section de cet article. À titre d'illustration, remarquons que le théorème 4 , appliqué aux structures conformes riemanniennes, conduit au :

COROLlaIRE 3. - Supposons qu'il existe un plongement conforme $\sigma$ d'une variété riemannienne $M$ plate, complète de dimension $n \geqslant 3$, dans une variété riemannienne $N$ de même dimension. Alors, ou bien $\sigma$ est un difféomorphisme conforme de $M$ sur $N$, ou bien $M$ est l'espace euclidien de dimension $n$, et $N$ est conformément équivalente à la sphère standard $\mathbf{S}^{n}$. De plus, $\sigma$ est la composée de la projection stéréographique standard, avec une transformation de Möbius.

$4^{\text {e }}$ SÉRIE - TOME $40-2007-\mathrm{N}^{\circ} 5$ 


\section{Un théorème de rigidité sur les plongements géométriques}

Cette dernière partie est consacrée à la preuve du théorème 4. Il s'agit en fait d'un cas particulier de rigidité des plongements géométriques de certaines géométries de Cartan, qui sera étudiée plus en détail dans [10].

\subsection{Plongements géométriques}

Soient $(M, B, \omega)$ et $\left(N, B^{\prime}, \omega^{\prime}\right)$, deux variétés munies de géométries de Cartan modelées sur un même espace $\mathbf{X}=G / P$ (on se place pour l'instant dans le cadre général : $G$ est un groupe de Lie et $P$ un sous-groupe fermé de $G$ ). Remarquons qu'en particulier, $M$ et $N$ ont même dimension.

On dira que $\sigma$ est un plongement géométrique de $M$ dans $N$ si $\sigma$ se relève en un plongement de fibrés $\hat{\sigma}: B \rightarrow B^{\prime}$, satisfaisant $\hat{\sigma}^{*} \omega^{\prime}=\omega$.

\subsection{Bord de Cauchy d'une géométrie de Cartan}

Nous décrivons ici une manière d'attacher un bord à toute géométrie de Cartan $(M, B, \omega)$, modelée sur un espace $\mathbf{X}=G / P$. Cette procédure a déjà été utilisée en relativité générale pour tenter d'associer un bord aux espaces temps, dans le but de mieux en comprendre les singularités (voir par exemple [22]).

Nous avons déjà vu en section 3 que le choix d'une base de $\mathfrak{g}$ définissait sur $B$ un parallélisme $\mathcal{R}$, et une métrique riemannienne $\rho$ qui rendait ce parallélisme orthonormé. Notons $d_{\rho}$ la distance associée à la métrique $\rho$. On peut considérer $(\bar{B}, \bar{d})$ le complété de Cauchy de l'espace métrique $\left(B, d_{\rho}\right)$. D'autre part, pour tout $b \in P$, la différentielle de $R_{b}$, lue dans la trivialisation de $T B$ fournie par le parallélisme $\mathcal{R}$, est l'application $A d\left(b^{-1}\right)$. Il en découle que, pour tout $b \in P$, $R_{b}$ est uniformément continue sur $\left(B, d_{\rho}\right)$, et qu'à ce titre, elle se prolonge en une application $\bar{R}_{b}$ sur $(\bar{B}, \bar{d})$. L'espace $\bar{B}$ s'écrit naturellement comme la réunion $\bar{B}=B \cup \partial_{c} B$. Le bord de Cauchy $\partial_{c} B$ est laissé stable par les transformations $\bar{R}_{b}, b \in P$. On pose alors $\partial_{c} M=\partial_{c} B / P$ (le quotient étant pris pour l'action de $P$ sur $\partial_{c} B$ via les transformations $\bar{R}_{b}$ ).

Bien entendu, en toute généralité, l'espace $M \cup \partial_{c} M=\bar{B} / P$ n'est pas séparé, ce qui confère à cette construction un intérêt limité pour obtenir de "jolis bords".

Voyons ce que donne cette construction dans le cas où $\mathbf{X}=\partial \mathbf{H}_{\mathbb{K}}^{d}$ :

LEMME 13. - (i) $\partial_{c} \mathbf{X}=\emptyset$.

(ii) $S i \Gamma \subset G$ est un sous-groupe discret qui agit proprement sur $\Omega_{\nu}$, le bord $\partial_{c}\left(\Gamma \backslash \Omega_{\nu}\right)$ est réduit à un point.

Preuve. - Rappelons que, pour l'espace modèle $\mathbf{X}$, le fibré de Cartan est juste le groupe $G$, et le choix d'une base de $\mathfrak{g}$ détermine une unique métrique $\rho_{G}$ invariante à gauche (et qui fait de la base choisie une base orthonormée). On note $B_{\nu}$ l'image réciproque de $\Omega_{o}$ pour la fibration $\pi_{X}: G \rightarrow \mathbf{X}$. Le bord topologique $\partial B_{\nu}$ de cet ouvert dans $G$ n'est autre que la fibre au-dessus de $o$. On note $\rho_{\nu}$ la métrique induite par $\rho_{G} \operatorname{sur} B_{\nu}$.

La variété riemannienne $\left(G, \rho_{G}\right)$ est homogène donc complète, ce qui prouve le point (i). Le groupe $\Gamma$ agit par isométries pour $\rho_{G}$. Ainsi, la variété $\Gamma \backslash G$ est munie d'une métrique $\bar{\rho}_{G}$, induite par $\rho_{G}$ via l'application de revêtement. La variété $\left(\Gamma \backslash G, \bar{\rho}_{G}\right)$ est également complète. Le quotient $\bar{B}_{\nu}=\Gamma \backslash B_{\nu}$ s'identifie à un ouvert de $\Gamma \backslash G$, et son bord topologique $\partial \bar{B}_{\nu} \subset \Gamma \backslash G$ est la sous-variété $\Gamma \backslash \partial B_{\nu}$. On appelle $\bar{\rho}_{\nu}$ la métrique induite par $\bar{\rho}_{G}$ sur $\bar{B}_{\nu}$. Comme $\partial \bar{B}_{\nu}$ est une sous-variété fermée de $\Gamma \backslash G$, il n'est pas difficile de vérifier que tout point $p_{\infty} \in \partial \bar{B}_{\nu}$ est limite d'une suite $\left(p_{k}\right)$ de $\bar{B}_{\nu}$, qui est de Cauchy pour la distance $d_{\bar{\rho}_{\nu}}$. Réciproquement, toute suite de $\bar{B}_{\nu}$ qui est de Cauchy pour $d_{\bar{\rho}_{\nu}}$ est a fortiori de Cauchy pour $d_{\bar{\rho}_{G}}$. Ainsi $\left(\Gamma \backslash G, d_{\bar{\rho}_{G}}\right)$ est le 
complété de Cauchy de $\left(\bar{B}_{\nu}, d_{\bar{\rho}_{\nu}}\right)$, et $\partial_{c} \bar{B}_{\nu}$ s'identifie à $\partial \bar{B}_{\nu}$. Comme l'action de $P$ est transitive sur $\partial \bar{B}_{\nu}$, on obtient bien que $\partial_{c}\left(\Gamma \backslash \Omega_{\nu}\right)$ est réduit à un point.

\subsection{Le bord géométrique d'un plongement}

Soient $(M, B, \omega)$ et $\left(N, B^{\prime}, \omega^{\prime}\right)$ deux géométries de Cartan modelées sur le même espace $\mathbf{X}=G / P$.

On suppose qu'il existe un plongement géométrique $\sigma: M \rightarrow N$. On appelle alors bord géométrique de $M$ associé au plongement $\sigma$, noté $\partial_{\sigma} M$, le bord topologique de $\sigma(M)$ dans $N$. De même, on notera $\partial_{\hat{\sigma}} B$ le bord topologique de $\hat{\sigma}(B)$ dans $B^{\prime}$.

On appelle $\rho$ et $\rho^{\prime}$ les métriques riemanniennes définies sur $B$ et $B^{\prime}$, associées au choix d'une même base de $\mathfrak{g}$, telles qu'elles ont été définies en section 3.1. Le plongement $\hat{\sigma}$ est alors en fait un plongement isométrique : $\hat{\sigma}^{*} \rho^{\prime}=\rho$. On note $d_{\rho}$ et $d_{\rho^{\prime}}$ les distances associées à $\rho$ et $\rho^{\prime}$.

DÉFINITION 12. - On appelle $d_{\rho^{\prime}}^{\sigma}$ la distance sur $\hat{\sigma}(B)$ définie par :

$$
d_{\rho^{\prime}}^{\sigma}(p, q)=\inf \left\{L_{\rho^{\prime}}(\gamma) \mid \gamma \in C^{1}([0,1], \hat{\sigma}(B)), \gamma(0)=p, \gamma(1)=q\right\} .
$$

Remarquons que $\hat{\sigma}$ est une isométrie de $\left(B, d_{\rho}\right)$ sur $\left(\hat{\sigma}(B), d_{\rho^{\prime}}^{\sigma}\right)$. En particulier, par équivariance de l'action de $P$, les transformations $R_{b}$, pour $b \in P$, sont uniformément continues pour la métrique $d_{\rho^{\prime}}^{\sigma}$.

On introduit également la :

DÉFINITION 13 (Points réguliers). - On dit qu'un point $p \in \partial_{\hat{\sigma}} B$ est régulier s'il existe une suite de points $\left(p_{k}\right)$ de $\hat{\sigma}(B)$ qui tend vers $p$, et telle que $\left(p_{k}\right)$ soit une suite de Cauchy pour la distance $d_{\rho^{\prime}}^{\sigma}$.

- On dit qu'un point $x \in \partial_{\sigma} M$ est régulier s'il existe un point régulier de $\partial_{\hat{\sigma}} B$ au-dessus de $x$.

- L'ensemble des points réguliers de $\partial_{\hat{\sigma}} B\left(\right.$ resp. $\left.\partial_{\sigma} M\right)$ est noté $\partial_{\hat{\sigma}}^{\text {reg }} B\left(\right.$ resp. $\left.\partial_{\sigma}^{\text {reg }} M\right)$.

Remarquons que comme $\partial_{\hat{\sigma}}^{\text {reg }} B$ est invariant par l'action de $P$ sur $B^{\prime}$, s'il existe un point de $\partial_{\hat{\sigma}}^{\mathrm{reg}} B$ au-dessus de $x$, alors tous les points de la fibre au-dessus de $x$ sont réguliers.

LEMME 14. - Si $\partial_{\sigma} M$ est non vide, alors $\partial_{\sigma}^{\mathrm{reg}} M$ est dense dans $\partial_{\sigma} M$.

Preuve. - On considère un point $x_{\infty} \in \partial_{\sigma} M$, et on le remonte en un point $\hat{x}_{\infty} \in \partial_{\hat{\sigma}} B$. On se fixe une petite boule $B_{\rho^{\prime}}\left(0_{\hat{x}_{\infty}}, \epsilon\right) \subset T_{\hat{x}_{\infty}} B^{\prime}$ telle que $\operatorname{Exp}_{\hat{x}_{\infty}}$ soit un difféomorphisme de $B_{\rho^{\prime}}\left(0_{\hat{x}_{\infty}}, \epsilon\right)$ sur son image. Pour tout $u \in B_{\rho^{\prime}}\left(0_{\hat{x}_{\infty}}, \epsilon\right)$, on appelle $T(u)$ l'ensemble des $t \in[0,1]$, pour lesquels il existe $\left.\left.b_{t} \in\right] t, 1\right]$, de sorte que $u_{0}^{*}(] t, b_{t}[)$ soit une composante connexe de $u_{0}^{*}(] 0,1[) \cap \hat{\sigma}(B)$. Pour chaque $u \in B_{\rho^{\prime}}\left(0_{\hat{x}_{\infty}}, \epsilon\right)$, on pose $\tau_{u}=\inf T_{u_{0}}$, avec la convention $\tau_{u}=+\infty$ si $T_{u}=\emptyset$. Remarquons que si $t \in T(u)$, alors $u^{*}(t)$ est un point régulier de $\partial_{\hat{\sigma}} B$. En effet, si $\left(t_{n}\right)$ est une suite de ] $t, b_{t}$ [ qui converge vers $t$, alors la suite $u^{*}\left(t_{n}\right)$ est de Cauchy pour $d_{\rho^{\prime}}{ }^{\sigma}$ et converge vers $u^{*}(t)$. Maintenant, on observe que $\operatorname{sinf}_{u \in B_{\rho^{\prime}}\left(0_{\left.\hat{x}_{\infty}, \epsilon\right)}\right.} \tau_{u}=\tau$ est strictement positif, cela signifie que $\operatorname{Exp}_{x_{\infty}}\left(B_{\rho^{\prime}}\left(0_{\hat{x}_{\infty}}, \tau\right)\right)$ ne rencontre pas $\sigma(M)$, une contradiction avec le fait que $x_{\infty} \in \partial_{\sigma} M$.

On en déduit qu'il existe une suite $\left(t_{i}\right)$ de $[0,1]$ qui tend vers 0 , avec $t_{i} \in T\left(u_{i}\right), u_{i} \in$ $B_{\rho^{\prime}}\left(0_{\hat{x}_{\infty}}, \epsilon\right)$ pour tout $i \in \mathbf{N}$. La suite $u_{i}^{*}\left(t_{i}\right)$ est alors une suite de points réguliers qui tend vers $\hat{x}_{\infty}$. En projetant, on récupère une suite de $\partial_{\sigma}^{\text {reg }} M$ qui converge vers $x_{\infty}$.

LEMME 15. - Il existe une application $\hat{\jmath}: \partial_{\hat{\sigma}}^{\text {reg }} B \rightarrow \mathcal{P}\left(\partial_{c} B\right)$ (où $\mathcal{P}\left(\partial_{c} B\right)$ désigne l'ensemble des parties de $\left.\partial_{c} B\right)$, telle que si $\hat{x}$ et $\hat{y}$ sont deux points distincts de $\partial_{\sigma}^{\mathrm{reg}} B$, alors $\hat{\jmath}(x) \cap \hat{\jmath}(y)=\emptyset$.

De plus, l'application $\hat{\jmath}$ est équivariante pour l'action de $P$ sur $\partial_{\hat{\sigma}}^{\text {reg }} B$ et $\mathcal{P}\left(\partial_{c} B\right)$. Elle induit donc une application $j: \partial_{\sigma}^{\text {reg }} M \rightarrow \mathcal{P}\left(\partial_{c} M\right)$ telle que $j(x) \cap j(y)=\emptyset$ lorsque $x \neq y$.

$4^{\text {e }}$ SÉRIE - TOME $40-2007-\mathrm{N}^{\circ} 5$ 
Preuve. - On définit l'application $\hat{\jmath}$ de la manière suivante : pour tout point $\hat{x}_{\infty} \in \partial_{\hat{\sigma}}^{\text {reg }} B$, $\hat{\jmath}\left(\hat{x}_{\infty}\right)$ est l'ensemble des suites $\left(\hat{x}_{k}\right)$ de $\hat{\sigma}(B)$ qui convergent vers $\hat{x}_{\infty}$ et qui sont de Cauchy pour $d_{\rho^{\prime}}{ }^{\sigma}$ (pour être précis, ce sont les suites $\hat{\sigma}^{-1}\left(\hat{x}_{k}\right)$ qui définissent des points de $\partial_{c} B$ ). Avec cette définition, si $b \in P$, il est clair que $\hat{\jmath}\left(R_{b} . \hat{x}_{\infty}\right)$ est la partie $R_{b} . \hat{\jmath}\left(\hat{x}_{\infty}\right)$, d'où l'équivariance de $\hat{\jmath}$. Si $\hat{x}_{\infty}$ et $\hat{y}_{\infty}$ sont distincts dans $\partial_{\hat{\sigma}}^{\text {reg }} B$, et que $\left(\hat{x}_{k}\right)$ et $\left(\hat{y}_{k}\right)$ sont deux suites de $\hat{\jmath}\left(\hat{x}_{\infty}\right)$ et $\hat{\jmath}\left(\hat{y}_{\infty}\right)$ respectivement, alors il existe $\epsilon>0$ tel que pour tout $k \in \mathbf{N}, d_{\rho^{\prime}}\left(\hat{x}_{k}, \hat{y}_{k}\right)>\epsilon$. Donc $a$ fortiori $d_{\rho^{\prime}}^{\sigma}\left(\hat{x}_{k}, \hat{y}_{k}\right)>\epsilon$, ce qui prouve que $\left(\hat{x}_{k}\right)$ et $\left(\hat{y}_{k}\right)$ sont distincts dans $\partial_{c} B$.

Remarquons que la propriété $\hat{\jmath}(\hat{x}) \cap \hat{\jmath}(\hat{y})=\emptyset$ pour $\hat{x} \neq \hat{y}$, couplée à la propriété d'équivariance implique que pour tout $\hat{x}_{\infty} \in \partial_{\hat{\sigma}}^{\text {reg }} B, \hat{\jmath}\left(\hat{x}_{\infty}\right)$ ne contient pas deux points qui sont sur une même $P$-orbite de $\partial_{c} B$. Ainsi, $\hat{\jmath}$ induit bien une application $j: \partial_{\sigma}^{\text {reg }} M \rightarrow \mathcal{P}\left(\partial_{c} M\right)$. Soient $x \neq y$ dans $\partial_{\sigma}^{\text {reg }} M$, et choisissons $\hat{x}$ et $\hat{y}$ au-dessus de $x$ et $y$ respectivement. Supposons que l'on ait $j(x) \cap j(y) \neq \emptyset$. Dans ce cas, $\hat{\jmath}(\hat{x}) \cap R_{b} .(\hat{\jmath}(\hat{y})) \neq \emptyset$, pour un certain $b \in P$. Par équivariance, cela implique que $\hat{\jmath}(\hat{x}) \cap \hat{\jmath}\left(R_{b} . \hat{y}\right) \neq \emptyset$, ce qui est impossible étant donné que $\hat{x} \neq R_{b} . \hat{y}$.

\subsection{Preuve du théorème 4}

On se place sous les hypothèses du théorème 4 , et on reprend les notations de la preuve du lemme 13.

Le premier cas à considérer est celui où $\partial_{\sigma}\left(\Gamma \backslash \Omega_{\nu}\right)$ est vide. Dans ce cas, $\sigma$ est un difféomorphisme. C'est un isomorphisme géométrique entre $\Gamma \backslash \Omega_{\nu}$ et $M$, et on est dans le cas (ii) du théorème.

Maintenant, supposons que $\partial_{\sigma}\left(\Gamma \backslash \Omega_{\nu}\right)$ soit non vide. Par le lemme 14, l'ensemble des points réguliers de $\partial_{\sigma}\left(\Gamma \backslash \Omega_{\nu}\right)$ est dense dans $\partial_{\sigma}\left(\Gamma \backslash \Omega_{\nu}\right)$, et en particulier, il est non vide. Mais le lemme 15 , associé au point $(i i)$ du lemme 13 , assure que l'ensemble des points réguliers est un singleton, qui de plus doit être dense dans $\partial_{\sigma}\left(\Gamma \backslash \Omega_{\nu}\right)$. Ceci prouve que $\partial_{\sigma}\left(\Gamma \backslash \Omega_{\nu}\right)$ est luimême réduit à un point que l'on appelle $\kappa$. Ainsi, $\partial_{\hat{\sigma}}^{\text {reg }} \bar{B}_{\nu}=\partial_{\hat{\sigma}} \bar{B}_{\nu}$ s'identifie à la fibre (i.e. une $P$-orbite) de $B$ au-dessus de $\kappa$. L'application $\hat{\jmath}$ du lemme 15 est une application équivariante de cette fibre sur $\partial_{c} \bar{B}_{\nu}$, ce dernier espace s'identifiant à $\Gamma \backslash P$. En particulier, l'action de $P$ sur $\Gamma \backslash P$ doit se faire sans point fixe, ce qui implique $\Gamma=\{e\}$.

Finalement, $M=\sigma\left(\Omega_{\nu}\right) \cup\{\kappa\}$. Ainsi, $M$ est difféomorphe à $\mathbf{X}$, donc simplement connexe, et d'autre part $(M, B, \omega)$ est plate puisqu'elle l'est sur un ouvert dense, et que sous l'hypothèse $\omega$ de classe $C^{1}$, la courbure est continue. On va avoir une application développante de $M$ dans $\mathbf{X}$, qui va être un revêtement, par compacité de $M$. L'application développante est alors un isomorphisme géométrique entre $M$ et $\mathbf{X}$.

\section{Remerciements}

Je voudrais remercier le rapporteur de cet article pour son précieux travail.

\section{RÉFÉRENCES}

[1] Biquard O., Métriques d'Einstein asymptotiquement symétriques, Astérisque 265 (2000).

[2] ČAP A., Two constructions with parabolic geometries, Rend. Circ. Mat. Palermo (2) Suppl. 79 (2006) 11-37.

[3] ČAP A., Schichl K., Parabolic geometries and canonical Cartan connections, Hokkaido Math. J. 29 (3) (2000) 453-505.

[4] CARTAN É., Les espaces généralisés, Notice sur les travaux scientifiques. Oeuvres Complètes, Partie I, vol. I, 1952, pp. 72-85.

[5] CARTAN É., Sur la géométrie pseudo-conforme des hypersurfaces de deux variables complexes I, Ann. Math. Pures Appl. 11 (4) (1932) 17-90. 
[6] CARTAn É., Sur la géométrie pseudo-conforme des hypersurfaces de deux variables complexes II, Ann. Scuola Norm. Sup. Pisa 1 (2) (1932) 333-354.

[7] Chern S.S., Moser J., Real hypersurfaces in complex manifolds, Acta Math. 133 (1974) 219-271.

[8] FERRAND J., Transformations conformes et quasi-conformes des variétés riemanniennes compactes, Mémoires Académie Royale de Belgique. Classe des Sciences 39 (5) (1971) 1-44.

[9] FERRAND J., The action of conformal transformations on a Riemannian manifold, Math. Ann. 304 (2) (1996) 277-291.

[10] Frances C., Un théorème de rigidité pour certains plongements de géométries de Cartan, en préparation.

[11] FRAnCES C., Sur les variétés lorentziennes dont le groupe conforme est essentiel, Math. Ann. 332 (1) (2005) 103-119.

[12] Frances C., TARquini C., Autour du théorème de Ferrand-Obata, Ann. Global Anal. Geom. 21 (1) (2002) 51-62.

[13] Goldman W.M., Complex Hyperbolic Geometry, Oxford Mathematical Monographs. Oxford Science Publications, The Clarendon Press, Oxford University Press, New York, 1999.

[14] Kamada H., NAyATAni S., Quaternionic analogue of $C R$ geometry, Séminaire de Théorie Spectrale et Géométrie de Grenoble 19 (2001) 41-52.

[15] KnAPP A.W., Lie Groups Beyond an Introduction, second ed., Progress in Mathematics, vol. 140, Birkhäuser, Boston, 2002.

[16] Kobayashi S., Transformation Groups in Differential Geometry, Springer-Verlag, 1972.

[17] KUIPER N., On conformally flat spaces in the large, Ann. of Math. 50 (1949) 916-924.

[18] Lafontaine J., The theorem of Lelong-Ferrand and Obata, in: Conformal Geometry, Bonn, 1985/1986, in : Aspects Math., vol. E12, Vieweg, Braunschweig, 1988, pp. 93-103.

[19] Lichnerowicz A., Sur les transformations conformes d'une variété riemannienne compacte, $C . R$. Acad. Sci. Paris 259 (1964) 697-700.

[20] Morimoto T., Geometric structures on filtered manifolds, Hokkaido Math. J. 22 (1993) 263-347.

[21] Овата M., The conjectures on conformal transformations of Riemannian manifolds, J. Differential Geometry 6 (1971/72) 247-258.

[22] SCHmidt B., A new definition of conformal and projective infinity of space-times, Comm. Math. Phys. 36 (1) (1974) 73-90.

[23] SCHOEn R., On the conformal and CR automorphism groups, Geom. Funct. Anal. 5 (2) (1995) 464 481.

[24] Sharpe R.W., Differential Geometry. Cartan's generalization of Klein's Erlangen program, Graduate Texts in Mathematics, vol. 166, Springer-Verlag, New York, 2000.

[25] Sternberg S., Lectures on Differential Geometry, Prentice-Hall, Inc., Englewood Cliffs, NJ, 1964.

[26] TANAKA N., On non-degenerate real hypersurfaces, graded Lie algebras, and Cartan connections, Japan J. Math. 2 (1976) 131-190.

[27] TANAKA N., On the equivalence problem associated with simple graded Lie algebras, Hokkaido Math. J. 8 (1979) 23-84.

[28] Thurston W., Three Dimensional Geometry and Topology. Vol. 1, Princeton University Press, 1997, Edited by Silvio Levy.

[29] Webster S.M., On the transformation group of a real hypersurface, Trans. Amer. Math. Soc. 231 (1977) 179-190.

(Manuscrit reçu le 13 septembre 2006; accepté, après révision, le 16 juillet 2007.)

\footnotetext{
Charles FRANCES

Laboratoire de Topologie et Dynamique,

Université Paris-Sud,

Bât. 430,

91405 Orsay, France

E-mail :Charles.Frances@math.u-psud.fr

$4^{\mathrm{e}}$ SÉRIE - TOME $40-2007-\mathrm{N}^{\circ} 5$
} 